\title{
TRIANGLES OF GROUPS
}

\author{
ANDREW CHERMAK
}

\begin{abstract}
Given a certain commutative diagram of groups and monomorphisms, does there necessarily exist a group in which the given diagram is essentially a diagram of subgroups and inclusions? In general, the answer is negative, but J. Corson, and Gersten and Stallings have shown that in the case of a "nonspherical triangle" of groups the answer is positive. This paper improves on these results by weakening the non-sphericality requirement.
\end{abstract}

\section{INTRODUCTION}

A triangle of groups is a commutative diagram $(T)$ of groups and inclusion maps, for which there is the following sort of picture.

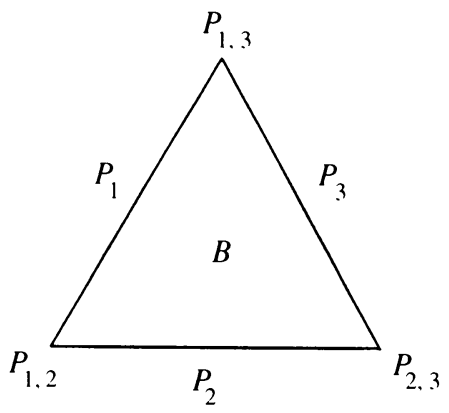

What we mean by this is that $B$ is a subgroup of each of the groups $P_{i}$, and that each $P_{i}$ and $P_{j}$ are subgroups of $P_{i, j}$. We shall always assume:

$$
P_{i} \cap P_{j}=B \text {, as subgroups of } P_{i, j},(1 \leq i \neq j \leq 3) \text {. }
$$

The triangle $(T)$ has an associated colimit group $G^{*}$, for which $(T)$ is essentially a presentation. Thus, there are natural homomorphisms from the groups $P_{i, j}$ into $G^{*}$, and one can ask whether these homomorphisms are injective. The answer "yes" means that $(T)$ is in fact a diagram of subgroups of some group, in which case we shall say that $(T)$ is realizable.

Sufficient conditions for the realizability of a triangle $(T)$ were given by Jon Corson in [5] and by Stallings in [11]. Namely, these authors showed that $(T)$ is realizable if $(T)$ is non-spherical in a sense which we shall now review. First, for any group $X$ and subgroup $Y$, let $Y \backslash X$ denote the set of right cosets of

Received by the editors May 24, 1993 and, in revised form, January 20, 1995.

1991 Mathematics Subject Classification. Primary 20E08, 20 F05. 
$Y$ in $X$. If $Y_{1}$ and $Y_{2}$ are subgroups of $X$ and $D$ is a subgroup of $Y_{1} \cap Y_{2}$ there is a graph

$$
\Gamma=\Gamma\left(X ; Y_{1}, Y_{2} ; D\right)
$$

whose vertex-set $V(\Gamma)$ and edge-set $E(\Gamma)$ are given by

$$
V(\Gamma)=\left(Y_{1} \backslash X\right) \amalg\left(Y_{2} \backslash X\right), \quad E(\Gamma) a=D \backslash X,
$$

and where the initial and terminal vertices of the edge $D g$ are $Y_{1} g$ and $Y_{2} g$, respectively. This graph is bipartite and admits a natural, edge-transitive action by $X$. The Stallings angle

$$
\theta=\theta(\Gamma)
$$

is defined to be $2 \pi / m$ where $m$ is the length of a shortest non-trivial cycle in $\Gamma$ (and where $m=\infty$ and $\theta=0$ if no such cycle exists).

Now, with $(T)$ a triangle of groups, there are graphs

$$
\Gamma_{i, j}=\Gamma\left(P_{i, j} ; P_{i}, P_{j}, B\right),
$$

and Stallings angles

$$
\theta_{i, j}=\theta\left(\Gamma_{i, j}\right) .
$$

In [5] and [11] a triangle is non-spherical if

$$
\theta_{1,2}+\theta_{1,3}+\theta_{2,3} \leq \pi
$$

and there is the following result.

(0.3) Theorem (Corson, Stallings). If $(T)$ is non-spherical in the sense of (0.2) then $(T)$ is realizable.

One aim of this paper is to improve on $(0.3)$ by weakening the criterion for non-sphericality. Thus, we shall show that $(T)$ is realizable even if each $\Gamma_{i, j}$ contains relatively short cycles (so that $(0.2)$ is violated), provided that these cycles do not fit together inside some larger geometric framework in certain prohibited ways. To this end, we shall employ the language of chamber systems (reviewed below in section 3 ).

Form the free amalgamated product

$$
G=P_{1,2} P_{2}^{*} P_{2,3},
$$

and form the rank-3 chamber system

$$
\mathscr{C}=\mathscr{C}\left(G, B,\left\{P_{1}, P_{2}, P_{3}\right\}\right) .
$$

The chambers of $\mathscr{C}$ are the right cosets of $B$ in $G$, and $\mathscr{C}$ consists of Cham $\mathscr{C}$ together with three equivalence relations (called $i$-adjacency), given by

$$
B x \sim_{i} B y \text { if } x y^{-1} \in P_{i} .
$$

Let $L$ be the subgroup of $G$ generated by $P_{1}$ and $P_{3}$ (regarded as subgroups of $G$ in the canonical way). By (0.1) we can make the identification:

$$
L=P_{1}{ }_{B} P_{3} .
$$

The inclusion maps of $P_{i}$ into $P_{1,3},(i=1,3)$, induce a homomorphism

$$
\zeta: L \rightarrow P_{1,3}
$$



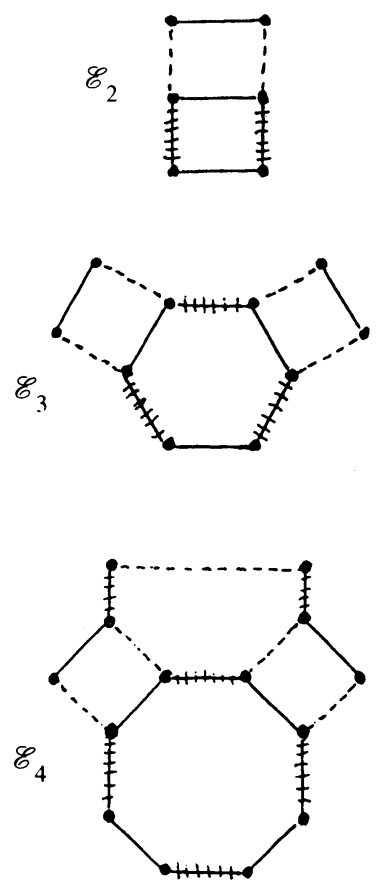

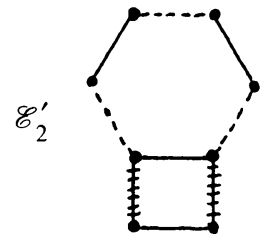

$\mathscr{E}_{3}^{\prime}$
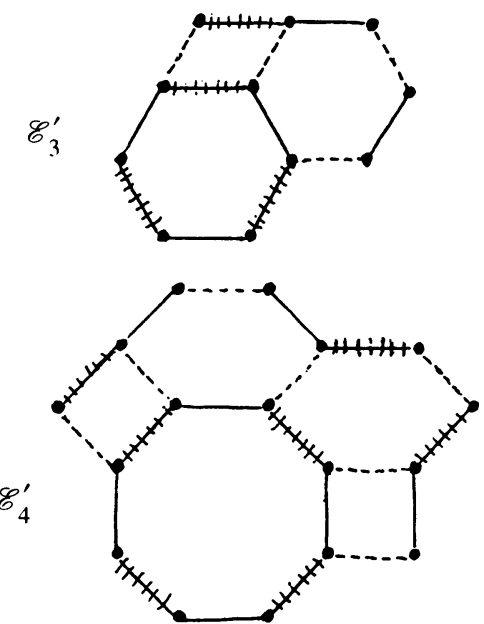

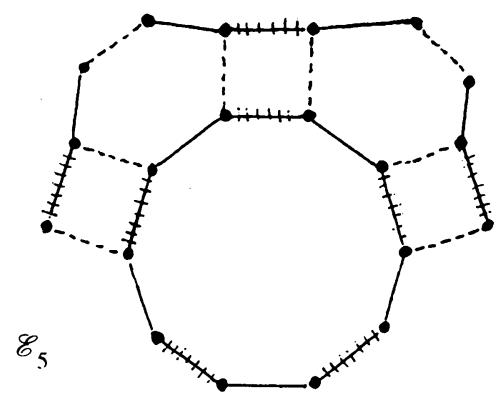

FIGURE 1 . The family $\mathbb{E}$

and we set

$$
K=\operatorname{ker}(\zeta) .
$$

For any $h \in K$ there is a quotient chamber system $\mathscr{C} /\langle h\rangle$. Our idea is to show that $(T)$ is realizable provided that each such quotient $\mathscr{C} /\langle h\rangle$ does not "involve" any of the chamber systems in a certain list $\mathbb{E}$, given in Figure 1. In more detail, we say that $\mathscr{C} /\langle h\rangle$ is $\mathbb{E}$-free if, for every $\mathscr{E} \in \mathbb{E}$, there exists no injective morphism $\mathscr{E} \rightarrow \mathscr{C} \mid\langle h\rangle$ of chamber systems over $\{1,2,3\}$. If this condition is satisfied we will call $(T)$ weakly non-spherical.

For any $\mathscr{E}$ in $\mathbb{E}$, the set Cham $\mathscr{E}$ of chambers of $\mathscr{E}$ is given by the set of "nodes" depicted in the diagram for $\mathscr{E}$ in Figure 1, while the $i$-adjacencies (other than the reflexive ones, which are implicit) are given by "edges" between 
nodes. A dotted edge is always a 2-adjacency, while the solid and hatched edges are to be read in two ways: as representing 1 and 3-adjacencies, respectively, or vice versa. Since the isomorphism classes of $\mathscr{E}_{1}$ and $\mathscr{E}_{4}^{\prime}$ are not altered by this $1-3$ polarity, $\mathbb{E}$ then consists of fourteen chamber systems over $\{1,2,3\}$.

Theorem A. Let $(T)$ be a triangle of groups (satisfying $(0.1)$ ), and let $\mathscr{C}$ and $K$ be as above. Assume that $\mathscr{C} /\langle h\rangle$ is $\mathbb{E}$-free for all $h \in K$. Then $(T)$ is realizable.

The proof of Theorem $\mathrm{A}$ is based on the arboreal small-cancellation theory developed in [3]. Thus, let $\mathbf{X}$ denote the standard tree for $G$, and put

$$
\mathscr{H}=\left(\bigcup_{g \in G}\left(g^{-1} K g\right)\right)-\{1\} .
$$

It turns out (see (2.1), below) that $\mathscr{H}$ is a set of hyperbolic isometries of $\mathbf{X}$. That is, for each $h \in \mathscr{H}$ there is a linear sub-tree $A(h)$ such that $h$ induces a translation on $A(h)$ of some positive amplitude $a(h)$. Let $\mathscr{H}_{n}^{*}$ denote the set of all finite $\mathscr{H}$-sequences $\mathbf{h}=\left(h_{0}, \ldots, h_{n}\right)$ such that $n+1$ is the minimal length for $h_{0} \cdots h_{n+1}$ as a word in $\mathscr{H}$. Put $\mathscr{H}^{*}=\bigcup\left\{\mathscr{H}_{n}^{*} \mid n \geq 0\right\}$. For each $n$ the Artin braid group on $n+1$ threads has a natural action on $\mathscr{H}_{n}^{*}$, generated by "simple braidings" of the form

$$
\left(h_{0}, \ldots, h_{i}, h_{i+1}, \ldots, h_{n}\right) \mapsto\left(h_{0}, \ldots, h_{i} h_{i+1} h_{i}^{-1}, h_{i}, \ldots, h_{n}\right) .
$$

0.4 Definition. Let $\mathscr{H}, \mathscr{H}^{*}$, and $\mathbf{X}$ be as above. We say that $\mathscr{H}$ has the convergence property (resp. the weak convergence property) if, whenever $\mathbf{h}^{\prime} \in \mathscr{H}_{n}^{*}$ and $u$ is a vertex of $\mathbf{X}$, there exists a braiding $\mathbf{h}=\left(h_{0}, \ldots, h_{n}\right)$ of $\mathbf{h}^{\prime}$ such that, upon setting $v=u h_{0} \cdots h_{n}$, the sequence of distances

$$
d(u, v), d\left(u h_{0}, v\right), d\left(u h_{0} h_{1}, v\right), \ldots, d\left(u h_{0} \cdots h_{n}, v\right)=0
$$

is monotonically decreasing (resp. non-increasing).

Theorem $\mathrm{A}$ is proved by showing that $\mathscr{H}$ has the weak convergence property if $\mathscr{C} /\langle h\rangle$ is $\mathbb{E}$-free for all $h \in K$.

If one thinks of $\mathbb{E}$ as detecting positive curvature, then the family $\mathbb{F}$ given in Figure 2 should be thought of as detecting non-negative curvature. We will prove the following results.

Theorem B. 1. If $\mathscr{C} /\langle h\rangle$ is $\mathbb{E}$-free for all $h \in K$, then $\mathscr{H}$ has the weak convergence property.

2. If $\mathscr{C} /\langle h\rangle$ is $\mathbb{F}$-free for all $h \in K$, then $\mathscr{H}$ has the convergence property.

In (2.4) and (2.6), below, we derive some consequences of convergence and of weak convergence. In particular, we find that $(T)$ is realizable, so each $P_{i, j}$ may be regarded as a subgroup of the colimit group $G^{*}$. Thus, there is a chamber system

$$
\mathscr{C}^{*}=\mathscr{C}\left(G^{*}, B,\left\{P_{1}, P_{2}, P_{3}\right\}\right)
$$

which can be identified with $\mathscr{C} /\langle\mathscr{H}\rangle$ if $\mathscr{C}^{*}$ is connected. We shall prove:

Theorem C. Assume that $\mathscr{C} /\langle h\rangle$ is $\mathbb{E}$-free (resp. $\mathbb{F}$-free) for all $h \in K$. Then the following hold.

(a) Any $\{i, j\}$-residue of $\mathscr{C}^{*}$ is isomorphic (as a graph) to a connected component of $\Gamma_{i, j},(1 \leq i \neq j \leq 3)$. 


$$
\mathscr{F}_{1}=\mathscr{E}_{1}
$$
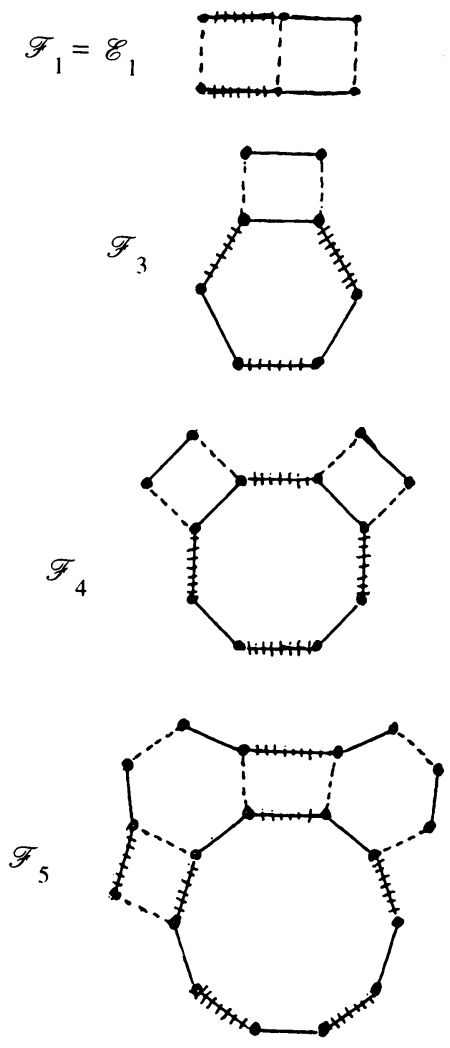
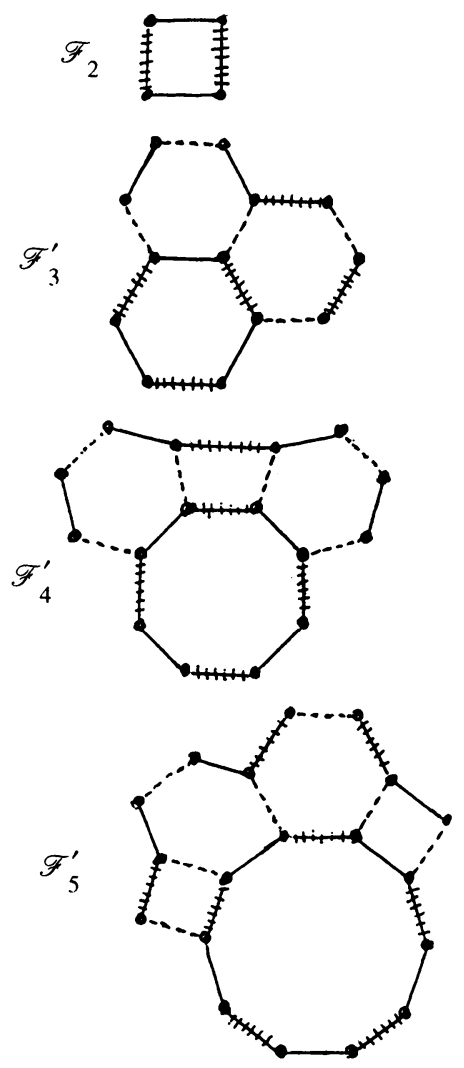

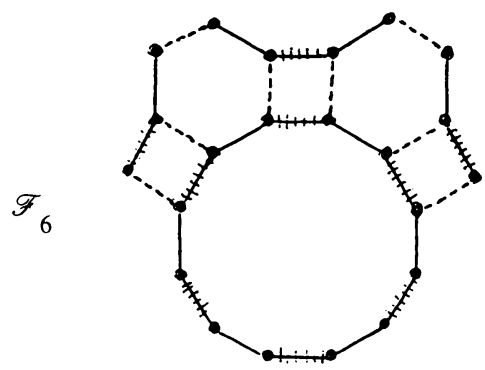

FIGURE 2. The family $\mathbb{F}$

(b) $\mathscr{C}^{*}$ is $\mathbb{E}$-free (resp. $\mathbb{F}$-free).

Our final result brings the discussion back to condition (0.2). Namely, suppose that $(T)$ is non-spherical in the Gersten-Stallings-Corson sense. Then $(T)$ is realizable (as those authors showed, and as a corollary also to Theorem A). With $G^{*}$ the colimit of $(T)$, as above, there are then graphs

$$
\Gamma_{i, j}^{*}=\Gamma\left(G^{*}, P_{i, k}, P_{j, k}, P_{k}\right)
$$

and Stallings angles

$$
\theta_{i, j}^{*}=\theta\left(\Gamma_{i, j}^{*}\right)
$$

for any ordering $(i, j, k)$ of $\{1,2,3\}$.

Theorem D. Suppose that $(T)$ is non-spherical in the sense of $(0.2)$. Then $\theta_{i, j}=$ $\theta_{i, j}^{*}$ for all $i \neq j$. 
The paper is organized as follows. Section 1 is a review of some basic material concerning tree-actions. In section 2 we discuss the action of $\mathscr{H}$ on $\mathbf{X}$ and derive consequences from a certain hypothesis $(2.3)$ which, later on (in sections 3 and 4), will be shown to be satisfied if $\mathscr{C} /\langle h\rangle$ is $\mathbb{E}$-free (or $\mathbb{F}$-free) for all $h \in K$. Section 3 begins with a review of chamber systems and establishes some useful connections between the tree $\mathbf{X}$ and the chamber system $\mathscr{C}$. Section 4 reviews material from [2] and contains the proof of Theorem B. The other theorems are obtained as corollaries in section 5.

Motivation. Our Theorem A is only a little bit better, apparently, than the corresponding result of Stallings, and of Corson, on non-spherical triangles. Moreover, our proofs are far lengthier than those of the above authors. The effort expended in weakening the "non-spherical" hypothesis to "almost non-spherical" will be justified provided that there exist interesting examples of almost nonspherical triangles which fail to be non-spherical. Such triangles are the subject of [4].

The triangles of groups considered in [4] arise in two quite different contexts. One of these concerns finite groups having a Sylow 2-subgroup isomorphic to a Sylow 2-subgroup of the simple group $\mathrm{CO}_{3}$ (the third Conway group). In 1974, Ronald Solomon investigated such groups, and he showed that $\mathrm{CO}_{3}$ is in fact the only finite simple group with such a Sylow 2-subgroup (of order $2^{10}$ ), [10]. But in the course of proving this result, Solomon produced a family of triangles of groups $\left(T_{p}\right)$ of the following type:

$\left(T_{p}\right)$

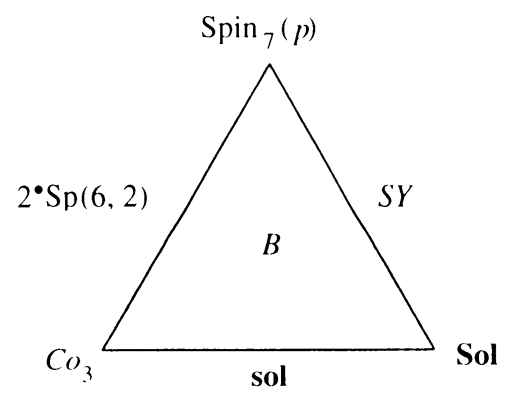

Here Sol is a (solvable) group of order $2^{10} \cdot 3^{4}$, sol is a maximal subgroup of $\mathrm{Co}_{3}$ of order $2^{10} \cdot 3^{3}$, and $\operatorname{Spin}_{7}(p)$ is the non-split double cover of the simple orthogonal group $\Omega_{7}(p), p$ an odd prime.

Much more recently, David Benson [2] has investigated an "exotic 2-adic finite loop space" discovered by Dwyer and Wilkerson [6]. In exploring the structure of this object, Benson demonstrates the existence of a triangle of groups (which I will call $\left(T_{*}\right)$ ) of the following type:

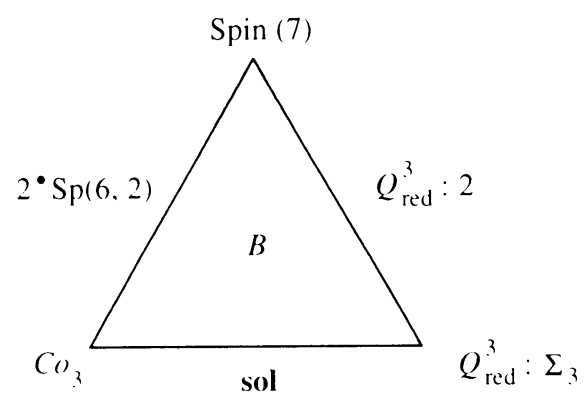


Here Spin (7) is the real, compact spin group in dimension 7, $Q$ denotes the group of unit-length quaternions, $Q^{3}$ the direct product of three copies of $Q$, and $Q_{\text {red }}^{3}=Q^{3} /\langle(-1,-1,-1)\rangle$. The semi-direct product $Q_{\text {red }}^{3}: \Sigma_{3}$ is induced by the natural permutation action of the symmetric group of degree 3 on $Q^{3}$.

It is not very difficult to show that colim $\left(T_{p}\right)=1$ for all odd primes $p$, and colim $\left(T_{*}\right)=1$. (The triviality of these colimits is a consequence of $[4$, Theorem 6.5]). Thus, $\left(T_{p}\right)$ and $\left(T_{*}\right)$ have "too many relations" for their own good. The main result of [4] is that there is a natural way to remove some relations, and to obtain a triangle $(\mathscr{J})$ which is almost non-spherical, but not non-spherical. Our Theorem A will then yield the existence of group $\mathscr{J}$ for which $(\mathscr{J})$ is a triangle of subgroups.

\section{ACTIONS ON TREES}

In this preliminary section we establish some notation and review some basic properties concerning groups acting on trees.

We view trees on the one hand as being combinatorial graphs. So we can speak of a pair of incident vertices $u$ and $v$ as forming an edge $(u, v)$, or of a sequence of vertices as forming a path $\left(u_{0}, \ldots, u_{n}\right)$ of length $n$. On the other hand, we feel free to identify any tree with its metric topological realization, for no other reason than to avail ourselves of a certain terminology. In particular, automorphisms of trees become isometries when trees are viewed in this way.

If $u$ and $v$ are vertices of a tree $\mathbf{X}$, then $[u, v]$ denotes the unique geodesic path from $u$ to $v$, also called the (directed) segment from $u$ to $v$. If $\sigma=$ $[u, v]$, then the length of $\sigma$ is denoted $\ell(\sigma)$, and is equal to the distance $d(u, v)$ from $u$ to $v$. A non-empty intersection of segments is again a segment, said to be degenerate if it is a single point.

An isometry of $\mathbf{X}$ is hyperbolic if it fixes no points in the topological realization of $\mathbf{X}$. Combinatorially, this means that the automorphism fixes no vertices and inverts no edges.

For any isometry $g$ of $\mathbf{X}$ we put

$$
\begin{aligned}
& a(g)=\operatorname{Inf}\{d(v, v g): v \in \mathbf{X}\}, \quad \text { and } \\
& A(g)=\{v \in \mathbf{X}: d(v, v g)=a(g)\} .
\end{aligned}
$$

(If $\mathbf{X}$ is viewed combinatorially then " $v \in \mathbf{X}$ " means that $v$ is either a vertex or an edge of $\mathbf{X}$.) We say that $a(g)$ is the amplitude of $g$, and observe that $g$ is hyperbolic if and only if $a(g)>0$. In this case $A(g)$ is called the axis of $g$.

We have the following fundamental result, due to Tits [12], and generalized to $\mathbb{R}$-trees by Morgan and Shalen [8], and to $\Lambda$-trees by Alperin and Bass. (See result 6.6 of [1].)

1.1 Lemma. Let $g$ be an isometry of a tree $\mathbf{X}$.

(a) If $[u, v]$ is an edge of $\mathbf{X}$ and $d(u, u g)=d(v, v g)$, then $[u, v]$ is an edge of $A(g)$.

(b) If $g$ is hyperbolic, then $A(g)$ is a "linear subtree" of $\mathbf{X}$; i.e. the image of an isometric embedding of $\mathbb{R}$ in $\mathbf{X}$. Moreover, $A(g)$ is the unique minimal $\langle g\rangle$-invariant subtree of $\mathbf{X}$ in this case.

(c) Let $u$ be a point of $\mathbf{X}$, and let $u_{0}$ be the point of $A(g)$ which is closest to $u$. Put $v=u g$ and $v_{0}=u_{0} g$. Then $\left[u_{0}, v_{0}\right]=[u, v] \cap A(g)$, and $\left[u_{0}, v_{0}\right]$ 
is centrally located in $[u, v]$, in the sense that $d\left(u, u_{0}\right)=d\left(v, v_{0}\right)$.

We shall only employ $(1.1)(c)$ in the case that $g$ is hyperbolic. In this case we get the following picture.

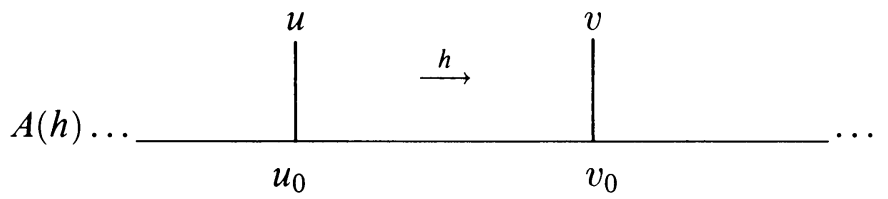

If $g$ is a hyperbolic isometry of $\mathbf{X},[u, v]$ is a segment of $A(g)$, and $w$ is a vertex of $\mathbf{X}$ with $v \in[u, w]$, then we say that $[u, v]$ is oriented towards $w$ by $g$ if $d(u g, v) \leq d(u, v g)$, and that $[u, v]$ is oriented away from $w$ by $g$ if $d(u g, v) \geq d(u, v g)$.

1.2 Lemma. Let $h$ and $h^{\prime}$ be hyperbolic isometries of a tree $\mathbf{X}$. Then the following two conditions are equivalent.

(1) $a\left(h h^{\prime}\right)<a(h)+a\left(h^{\prime}\right)$.

(2) $A(h) \cap A\left(h^{\prime}\right)$ contains a non-degenerate segment oriented in opposite directions by $h$ and $h^{\prime}$.

Proof. Choose a vertex $u$ of $A\left(h h^{\prime}\right)$ with $u$ as close as possible to $A(h) \cup A\left(h^{\prime}\right)$. Since $A\left(h h^{\prime}\right)=A\left(\left(h h^{\prime}\right)^{-1}\right)$, we may assume without loss of generality that

$$
d(u, A(h)) \leq d\left(u, A\left(h^{\prime}\right)\right) \text {. }
$$

Let $u_{0}$ be the vertex of $A(h)$ nearest to $u$ and put $v_{0}=u_{0} \cdot h, v=u \cdot h$, $d=d\left(u, u_{0}\right)$. Then $d(u, v)=a(h)+2 d$, and $\left[u_{0}, v_{0}\right]$ is centrally located in $[u, v]$, by $(1.1)(\mathrm{c})$.

Put $w=v \cdot h^{\prime}$, and let $y$ denote the vertex of $[u, v]$ which is closest to $w$. Suppose first that $y$ is in the interval $\left[v_{0}, v\right]$. We then have

$$
\begin{aligned}
a\left(h h^{\prime}\right) & =d(u, w) \\
& =d(u, y)+d(y, w) \\
& \geq d\left(u, v_{0}\right)+d(v, w)-d(v, y) \\
& \geq d+a(h)+d(v, w)-d \\
& =a(h)+a\left(h^{\prime}\right) .
\end{aligned}
$$

Thus, condition (1), above, fails to hold in this case.

Assume that (2) fails to hold, and put $J=A\left(h^{\prime}\right) \cap[u, v]$. As $[y, v]$ is oriented towards $y$ by $h^{\prime}$ it then follows from $(*)$ that either $J$ is degenerate or $J \subseteq\left[v_{0}, v\right]$. Let $d^{\prime}$ denote the distance from $v$ to $J$. Assume further that $y$ is in the interval $\left[u, v_{0}\right)$. If $J$ is degenerate we have $J \subseteq\{y\}$ and $d\left(y, v_{0}\right) \leq d(y, v) \leq d^{\prime}$. If $J$ is non-degenerate, then $\ell(J)=a\left(h^{\prime}\right)$ as otherwise $(1.1)$ (c) would put $y$ elsewhere than in $\left[u, v_{0}\right)$. In this case we have $d\left(y, v_{0}\right) \leq d\left(w, v_{0}\right) \leq d^{\prime}$, so $d\left(y, v_{0}\right) \leq d^{\prime}$ in either case. On the other hand, we have

$$
\begin{aligned}
a\left(h h^{\prime}\right) & =d(u, w) \\
& =d(u, y)+d(y, w) \\
& =d(u, v)-d(y, v)+d(y, w) \\
& =d(u, v)-2 d(y, v)+d(v, w) \\
& =a(h)+a\left(h^{\prime}\right)+2 d+2 d^{\prime}-2 \cdot d(y, v) \\
& =a(h)+a\left(h^{\prime}\right)+2 d^{\prime}-2 \cdot d\left(y, v_{0}\right),
\end{aligned}
$$


so we conclude that $a\left(h h^{\prime}\right) \geq a(h)+a\left(h^{\prime}\right)$.

This proves that (1) implies (2). The reverse implication is obtained much more easily, and we leave this simple verification to the reader.

\section{TREES, GRAPHS, AND CONVERGENCE}

For the remainder of this paper $(T)$ will denote a triangle of groups, labelled as on the first page of the Introduction, and satisfying (0.1). Fix the following notation:

$$
\begin{aligned}
G & =P_{1,2} \underset{P_{2}}{*} P_{2,3}, \\
L & =P_{1} \underset{B}{*} P_{3}, \\
K & =\operatorname{ker}\left(L \rightarrow P_{1,3}\right) \quad(\text { the kernel of the canonical homomorphism), } \\
\mathbf{X} & =\Gamma\left(G ; P_{1,2}, P_{2,3} ; P_{2}\right), \quad \text { and } \\
\mathbf{Y} & =\Gamma\left(L ; P_{1}, P_{3} ; B\right) .
\end{aligned}
$$

Regard $P_{1}$ and $P_{3}$ as subgroups of $G$ via $P_{i} \subseteq P_{i, 2} \subseteq G$, and let $L^{*}$ denote the subgroup of $G$ generated by $P_{1}$ and $P_{3}$. There is then a natural epimorphism $\xi$ of $L$ onto $L^{*}$. Now define a mapping

$$
\delta: \mathbf{Y} \rightarrow \mathbf{X}
$$

by $\delta(B g)=P_{2} \cdot \xi(g)$ and $\delta\left(P_{i} g\right)=P_{i, 2} \cdot \xi(g)$ for $i=1$ and $3, g \in L$. One easily verifies that $\delta$ is a $\xi$-equivariant morphism of graphs.

2.0 Lemma. $\delta$ is injective, and $\xi$ is an isomorphism.

Proof. For the injectivity of $\delta$ it suffices, since $\mathbf{X}$ is a tree, to show that $\delta$ is locally injective. Then, since $L$ is transitive on the edges of $\mathbf{Y}$, it suffices to show that $\delta$ restricted to $B \backslash P_{i}$ is injective $(i=1,3)$. If not, then for some $i, i=1$ or 3 , there exists $g \in P_{i}$ such that $B \neq B g$ and $P_{2}=P_{2} \cdot \xi(g)$. Since $P_{i} \subseteq P_{i, 2} \subseteq G$, the restriction of $\xi$ to $P_{i}$ is just the inclusion map from $P_{i}$ into $G$. Thus, $P_{2}=P_{2} g$. But then $g \in P_{i} \cap P_{2}=B$, after all, by $(0.1)$, so $\delta$ is injective. Now let $g$ be an element of $\operatorname{ker}(\xi)$. As $\delta$ is $\xi$-equivariant, $g$ acts trivially on $\mathbf{Y}$. Then $g \in B$, and since $\xi \mid B$ is injective we get $g=1$. Thus, $\xi$ is an isomorphism. $L^{*}$.

Henceforth, we shall simply identify $\mathbf{Y}$ with the image of $\delta$, and $L$ with

2.1 Corollary. Regard $P_{1,2}, P_{2,3}$, and $L$ as subgroups of $G$ in the canonical way. Then, as subgroups of $G$, we have

(a) $P_{i} \cap P_{j}=B,(1 \leq i \neq j \leq 3)$,

(b) $P_{1,2} \cap P_{2,3}=P_{2}$, and

(c) $L \cap P_{i, 2}=P_{i},(i=1,3)$.

We now set

$$
\mathscr{H}=\left(\bigcup_{g \in G} g^{-1} K g\right)-\{1\}
$$


2.2 Lemma. $\mathscr{H}$ is a symmetric, self-normalizing set of hyperbolic isometries of $\mathbf{X}$. Moreover, $a(h) \geq 4$ for all $h \in \mathscr{H}$.

Proof. By construction we have $\mathscr{H}=\mathscr{H}^{-1}$ (so $\mathscr{H}$ is symmetric) and $g^{-1 \mathscr{H} g}$ $=\mathscr{H}$ for all $g \in G$. In particular, $h^{-1} \mathscr{H} h=\mathscr{H}$ for all $h \in \mathscr{H}$ (so $\mathscr{H}$ is self-normalizing). Put $\mathscr{K}=K-\{1\}$.

Recall that $(T)$ provides inclusions

$$
B \subseteq P_{1} \subseteq P_{1,3} \text { and } B \subseteq P_{3} \subseteq P_{1,3},
$$

and that $K$ is the kernel of the homomorphism $L \rightarrow P_{1,3}$ induced by these inclusions. Regarding $P_{1}$ and $P_{3}$ as subgroups also of $L$ we then have $K \cap P_{1}=$ $K \cap P_{3}=1$. Since $K$ is normal, this shows that $\mathscr{K}$ is a set of hyperbolic isometries of $\mathbf{Y}$, and then $(1.1)(b)$ says that each element $h$ of $\mathscr{K}$ has an axis $A(h)$ in $\mathbf{Y}$, on which $h$ acts as a translation of amplitude $a(h)$. Regarding $\mathbf{Y}$ as a subtree of $\mathbf{X},(1.1)(\mathrm{a})$ shows that $A(h)$ is also the axis of $h$ in $\mathbf{X}$, and $h$ is a hyperbolic isometry of $\mathbf{X}$. Then $\mathscr{H}$ is a set of hyperbolic isometries of $\mathbf{X}$, as desired. Further, since $\mathbf{Y} / K$ contains no cycles of length 2 , by $(0.1)$, it follows that $a(h) \neq 2$ for any $h \in \mathscr{H}$. But $a(g)$ is even for any $g \in G$ since $G$ preserves the bipartite structure of $\mathbf{X}$. This completes the proof of (2.2).

The reader will need to recall definition $(0.4)$.

\subsection{Hypothesis. One of the following holds.}

(a) $\mathscr{H}$ has the weak convergence property, and $\ell(\mathbf{Y} \cap A(h))<\frac{1}{2} a(h)$ for all $h \in \mathscr{H}-K$.

(b) $\mathscr{H}$ has the convergence property, and $\ell(\mathbf{Y} \cap A(h)) \leq \frac{1}{2} a(h)$ for all $h \in$ $\mathscr{H}-K$.

We assume Hypothesis (2.3) for the remainder of this section.

Let $g \in\langle\mathscr{H}\rangle$, let $u$ be a vertex of $\mathbf{X}$, and let $\mathbf{h}=\left(h_{0}, \ldots, h_{n}\right)$ be a sequence of elements of $\mathscr{H}$. We will say that $\mathbf{h}$ is a $(g, u)$-sequence if

(i) $\mathbf{h}$ is a word of minimal length for $g$ in terms of $\mathscr{H}$, and

(ii) setting $v=u g$, the sequence of distances

$$
d(u, v), d\left(u h_{0}, v\right), \ldots, d\left(u h_{0} \cdots h_{n}, v\right)=0
$$

is monotonically non-increasing, and is monotonically decreasing if $(2.3)(\mathrm{b})$ holds.

It follows from Hypothesis (2.3) and definition (0.4) that, for any $g \in\langle\mathscr{H}\rangle$ and vertex $u$ of $\mathbf{X}$, there always exists at least one $(g, u)$-sequence.

2.4 Theorem. Assume Hypothesis (2.3). Then

(a) $\mathbf{Y} \cap \mathbf{Y g}=\varnothing$ for all $g \in\langle\mathscr{H}\rangle-K$.

(b) $\langle\mathscr{H}\rangle$ is a free group acting freely on $\mathbf{X}$, and $\langle\mathscr{H}\rangle \cap L=K$.

(c) $(T)$ is realizable.

(d) Let $\bar{L}$ be the subgroup of $P_{1,3}$ generated by $P_{1}$ and $P_{3}$, and identify $\bar{L}$ with $L\langle\mathscr{H}\rangle /\langle\mathscr{H}\rangle$ via $(\mathrm{b})$, above. Then the colimit of $(T)$ is given by the free amalgamated product

$$
G /\langle\mathscr{H}\rangle_{\frac{*}{L}} P_{1,3} .
$$

Proof. Suppose that (a) is false. Then there is a vertex $u$ of $\mathbf{Y}$ and an element $g$ of $\langle\mathscr{H}\rangle-K$ such that $u g \in V(\mathbf{Y})$. Among all such $g$ and $u$, choose 
these two so that the length of $g$ as a word in $\mathscr{H}$ is as small as possible. Let $\mathbf{h}=\left(h_{0}, \ldots, h_{n}\right)$ be a $(g, u)$-sequence. We then have

$$
d(u, u g) \begin{cases}\geq d\left(u h_{0}, u g\right) & \text { if }(2.3)(\mathrm{a}), \\ >d\left(u h_{0}, u g\right) & \text { if }(2.3)(\mathrm{b}) .\end{cases}
$$

The reader may now wish to refer to the diagram introduced in the proof of (1.1), with $h_{0}$ in the role of $h$. Let $x$ be the vertex of $A\left(h_{0}\right)$ which is nearest to $u g$. It follows that $d(u, x) \geq d\left(u h_{0}, x\right)$ (strict if (2.3)(b)). Let $u_{0}$ and $v_{0}$ be as in the diagram. We then have $d\left(u_{0}, x\right) \geq d\left(v_{0}, x\right)$ ( $>$ if $\left.(2.3)(\mathbf{b})\right)$, and so

$$
\ell\left([u, u g] \cap A\left(h_{0}\right)\right) \begin{cases}\geq \frac{1}{2} a\left(h_{0}\right) & \text { if }(2.3)(\mathbf{a}), \\ >\frac{1}{2} a\left(h_{0}\right) & \text { if }(2.3)(\mathbf{b}) .\end{cases}
$$

As $[u, u g] \subseteq \mathbf{Y}$ by assumption, we now conclude from $(2.3)(\mathbf{a})$ or (b) that $h_{0} \in \mathscr{K}$. Set $g^{\prime}=h_{0}^{-1} g$ and set $u^{\prime}=u h_{0}$. Then $u^{\prime} \in V(\mathbf{Y})$ since $\mathbf{Y}$ is $K$-invariant. Also, we have $g^{\prime} \in\langle\mathscr{H}\rangle, u^{\prime} g^{\prime}=u g \in V(\mathbf{Y})$, and the length of $g^{\prime}$ as a word in $\mathscr{H}$ is less than that of $g$. The minimality condition on $g$ then implies that $g^{\prime} \in K$, and since $K$ is a group we get $g=h_{0} g^{\prime} \in K$, for a contradiction. This proves (a).

Since any vertex of $\mathscr{Z}$ lies in a $G$-translate of $\mathbf{Y}$, it follows immediately from (a) that $\langle\mathscr{H}\rangle$ acts freely on $\mathbf{X}$ and that $\langle\mathscr{H}\rangle \cap L=K$. Free groups are just groups which act freely on simplicial trees, so (b) is now proven.

Set $G_{0}=G /\langle\mathscr{C}\rangle$ and observe that, by (b), there are natural injective mappings from $P_{1,2}, P_{2,3}$, and $L / K$ into $G_{0}$. Let $\bar{L}$ denote the subgroup of $P_{1,3}$ generated by $P_{1}$ and $P_{3}$. Then $L / K \cong \bar{L}$ and we may identify the colimit group of $(T)$ as

This proves (c), and (d).

$$
\operatorname{colim}(T)=G_{0} \frac{*}{L} P_{1,3}
$$

For the remainder of this section let $G^{*}$ be the colimit group of $(T)$, in the form given by

$$
G^{*}=(G /\langle\mathscr{H}\rangle) \frac{*}{L} P_{1,3},
$$

as in $(2.4)(\mathrm{d})$. Our aim will now be to obtain information concerning the Stallings angles associated with $(T)$ and with $G^{*}$, with a view towards the proof of Theorem D.

For any ordering $(i, j, k)$ of $\{1,2,3\}$, put

$$
\begin{aligned}
& \Gamma_{i, j}=\Gamma\left(P_{i, j} ; P_{i}, P_{j} ; B\right), \quad \text { and } \\
& \Gamma_{i, j}^{*}=\Gamma\left(G^{*} ; P_{i, k}, P_{j, k} ; P_{k}\right),
\end{aligned}
$$

as in the Introduction. Let $m_{i, j}$ be the length of a shortest non-trivial cycle in $\Gamma_{i, j}$, taking $m_{i, j}=\infty$ if $\Gamma_{i, j}$ is a forest. Similarly define $m_{i, j}^{*}$ with respect to $\Gamma_{i, j}^{*}$. The Stallings angles associated with $\Gamma_{i, j}$ and $\Gamma_{i, j}^{*}$ are then

$$
\theta_{i, j}=2 \pi / m_{i, j} \text { and } \theta_{i, j}^{*}=2 \pi / m_{i, j}^{*},
$$

respectively. Finally, define morphisms of graphs,

$$
\delta_{i, j}: \Gamma_{i, j} \rightarrow \Gamma_{i, j}^{*}
$$

by $B g \mapsto P_{k} g, P_{i} g \mapsto P_{i, k} g$, and $P_{j} g \mapsto P_{j, k} g$ for all $g \in P_{i, j}$. 
2.5 Theorem. Assume Hypothesis (2.3), and let notation be as above. Then the following hold.

(a) Within $G^{*}$, we have $P_{i} \cap P_{j}=B$ and $P_{i, j} \cap P_{j, k}=P_{j}$ for all orderings $(i, j, k)$ of $\{1,2,3\}$.

(b) For all $i \neq j$, the morphism

$$
\delta_{i, j}: \Gamma_{i, j} \rightarrow \Gamma_{i, j}^{*}
$$

is injective.

(c) Let $\gamma^{*}$ be a cycle (without back-tracking) of minimal length in $\Gamma_{1,3}^{*}$. Then there exists a cycle $\gamma$ in $\Gamma_{1,3}$ such that $\gamma^{*}$ is a translation of $\delta_{1,3}(\gamma)$ by an element of $G^{*}$.

(d) $\theta_{1,3}=\theta_{1,3}^{*}$, and $\theta_{i, j} \leq \theta_{i, j}^{*}$ for all $i \neq j$.

Proof. Let $M$ be a subgroup of $L, N$ a subgroup of $P_{i, 2}$ for some $i,(i=1$ or 3 ), and $s$ an element of $M\langle\mathscr{H}\rangle \cap N$. Write $s=x g$ with $x \in M$ and $g \in\langle\mathscr{H}\rangle$. Now let $u$ be the vertex $P_{i, 2}$ of $\mathbf{X}$ and put $v=u x$. Then $v g=u s=u$, so both $v$ and $v g$ are vertices of $\mathbf{Y}$. Then $g \in K$ by (2.4)(a), so $M\langle\mathscr{H}\rangle \cap N=M K \cap N$.

Apply the above result with $M=P_{i}$ and $N=P_{2}$, obtaining

$$
P_{i}\langle\mathscr{H}\rangle \cap P_{2}=P_{i} K \cap P_{2} \subseteq L \cap P_{2}=B,
$$

by way of $(2.1)(\mathrm{c})$ and $(2.1)(\mathrm{a})$. Thus, in $G^{*}$ we have $P_{i} \cap P_{2}=B,(i=1,3)$. Taking $M=P_{1}$ and $N=P_{3}$ we also get

$$
P_{1}\langle\mathscr{H}\rangle \cap P_{3}=P_{1} K \cap P_{3} .
$$

But $P_{1} K \cap P_{3}=B$, by $(0.1)$, so we conclude that in $G^{*}$ also, $P_{1} \cap P_{3}=B$.

Next, taking $M=L$ and $N=P_{i, 2}$, we obtain $L\langle\mathscr{H}\rangle \cap P_{i, 2}=L \cap P_{i, 2}=P_{i}$ by $(2.1)(\mathrm{c})$, so $\bar{L} \cap P_{i, 2}=P_{i}$ in $G^{*}$. Then also $P_{1,3} \cap P_{i, 2}=P_{i}$ in $G^{*}$ by $(2.4)(\mathrm{d})$. In order to complete the proof of (a) we now need only show that $P_{1,2}\langle\mathscr{H}\rangle \cap P_{2,3}=P_{2}$ in $G$.

Let now $x \in P_{1,2}$ and $g \in\langle\mathscr{H}\rangle$ with $t=x g \in P_{2,3}$. Suppose $g \neq 1$. We recall from $(2.2)$ that $a(h) \geq 4$ for all $h \in \mathscr{H}$, and then since $\mathscr{H}$ has the weak convergence property it follows that also $a(g) \geq 4$. On the other hand $g$ sends the vertex $P_{1,2} t^{-1}$ to $P_{1,2} t^{-1} g=P_{1,2} x^{-1}=P_{12}$, and the distance from $P_{1,2}$ to $P_{1,2} t^{-1}$ is at most 2 since $\left(P_{1,2} t^{-1}, P_{2,3}, P_{1,2}\right)$ is a path in $\mathbf{X}$. Thus, $a(g) \leq 2$. This contradiction shows $g=1$, and so $P_{1,2}\langle\mathscr{H}\rangle \cap P_{2,3}=$ $P_{1,2} \cap P_{2,3}=P_{2}$, as desired. This proves (a).

Part (b) is an immediate consequence of (a), and (b) implies $\theta_{i, j} \leq \theta_{i, j}^{*}$ for all $i \neq j$. Since (c) implies $\theta_{1,3}=\theta_{1,3}^{*}$ it now only remains to prove $(\mathrm{c})$.

Set $\Gamma=\Gamma_{1,3}, \Gamma^{*}=\Gamma_{1,3}^{*}$, and $\delta=\delta_{1,3}$. Let $\gamma^{*}$ be a cycle of minimal length (of length $\ell$, say) in $\Gamma^{*}$. Let $\Gamma_{0}^{*}$ be the connected component of $\Gamma^{*}$ containing the vertices $P_{1,2}$ and $P_{2,3}$, and identify $\Gamma_{0}^{*}$ with $\mathbf{X} /\langle\mathscr{H}\rangle$. Similarly, the connected component of $\Gamma$ containing the vertices $P_{1}$ and $P_{3}$ may be identified with $\mathbf{Y} / K$. Without loss of generality, we may assume that $\gamma^{*}$ is a cycle in $\Gamma_{0}^{*}$.

As $\mathbf{X}$ is the universal cover of $\Gamma_{0}^{*}$, there is a linear subtree $A$ of $\mathbf{X}$ which projects onto $\gamma^{*}$, and the minimality of $\ell$ implies that $A=A(g)$ for some $g \in\langle\mathscr{H}\rangle$, with $a(g)=\ell$. 
Let $u$ be a vertex of $A$, and let $\mathbf{h}=\left(h_{0}, \ldots, h_{n}\right)$ by a $(g, u)$-sequence. Put $v=u g$, set $u_{0}=u$, and define $u_{1}, \ldots, u_{n}$ by $u_{i+1}=u_{i} h_{i}, 0 \leq i \leq n$. Then $v=u_{n+1}$ and we have

$$
\ell=d\left(u_{0}, v\right) \geq d\left(u_{1}, v\right) \geq \cdots \geq d\left(u_{n+1}, v\right)=0,
$$

with strict inequality if $(2.3)$ (b) holds.

If $n=0$, then $g=h_{0}$ lies in a conjugate of $K, A$ is contained in a $G$ translate of $\mathbf{Y}$, and $\gamma^{*}$ is a $G^{*}$-translate of $\delta(\gamma)$ where $\gamma$ is the corresponding image of $A$ in $\mathbf{Y} / K$. That is, the conclusion of (c) holds in this case, so we may assume $n \geq 1$. Then $\left[u_{n}, v\right]$ contains a segment of $A\left(h_{n}\right)$ of length $a\left(h_{n}\right)$. Such a segment projects to a cycle in $\mathbf{X} /\langle\mathscr{H}\rangle$, so the minimality of $\ell$ implies that $d\left(u_{n}, v\right)=a\left(h_{n}\right)=\ell$. Thus,

$$
\ell=d\left(u_{0}, v\right)=d\left(u_{1}, v\right)=\cdots=d\left(u_{n}, v\right) .
$$

In particular, we are in the situation of $(2.3)(a)$.

Now $\left[u_{n-1}, u_{n}\right]$ is a segment of $A\left(h_{n-1}\right)$, again by the minimality of $\ell$. It now follows from $(*)$ that $\left[u_{n-1}, u_{n}\right]$ and $\left[u_{n}, v\right]$ intersect in a segment of length $\frac{1}{2} \ell$. But $(2.3)(\mathrm{a})$ then implies that $h_{n-1}$ and $h_{n}$ lie in a common conjugate of $K$. Thus $h_{n-1} h_{n} \in \mathscr{H} \cup\{1\}$, and $\mathbf{h}$ is not a minimal-length word for $g$. This contradiction completes the proof of (2.5).

We next consider the graphs

$$
\begin{gathered}
\tilde{\Gamma}_{1,2}=\Gamma\left(G ; L, P_{2,3} ; P_{3}\right), \quad \text { and } \\
\tilde{\Gamma}_{2,3}=\Gamma\left(G ; L, P_{1,2} ; P_{1}\right) .
\end{gathered}
$$

Notice that there are natural surjective morphisms of graphs $\tilde{\Gamma}_{1,2} \rightarrow \Gamma_{1,2}^{*}$ and $\tilde{\Gamma}_{2,3} \rightarrow \Gamma_{2,3}^{*}$. Also, there are natural morphisms

$$
\psi_{1,2}: \Gamma_{1,2} \rightarrow \tilde{\Gamma}_{1,2}, \quad \psi_{2,3}: \Gamma_{2,3} \rightarrow \tilde{\Gamma}_{2,3} .
$$

These are defined in the usual way. For example, $\psi_{2,3}$ is defined by

$$
\psi_{2,3}\left\{\begin{aligned}
B g & \mapsto P_{1} g \\
P_{2} g & \mapsto P_{1,2} g \\
P_{3} g & \mapsto L
\end{aligned}\right.
$$

for $g \in P_{2,3}$. We will need the following result.

2.6 Lemma. For $k=1$ and 3 , let $\psi_{2, k}: \Gamma_{2, k} \rightarrow \tilde{\Gamma}_{2, k}$ be defined as above. Then $\psi_{2, k}$ is injective. Moreover, if $\tilde{\gamma}$ is a cycle in $\tilde{\Gamma}_{2, k}$, then there exists a cycle $\gamma$ in $\Gamma_{2, k}$ such that $\tilde{\gamma}$ is a translation of $\psi_{2, k}(\gamma)$ by an element of $G$.

Proof. Without loss we may take $k=3$. We note that (2.1) yields $P_{1} \cap P_{2,3}=$ $B, P_{1,2} \cap P_{2,3}=P_{2}$, and $L \cap P_{2,3}=P_{3}$, where all these intersections are taken in $G$. It follows that $\psi_{2,3}$ is injective.

Let $\tilde{\gamma}$ be a cycle in $\tilde{\Gamma}_{2,3}$, without back-tracking. Then $\tilde{\gamma}$ may be viewed (upon first translating by a suitable element of $G$ ) as a word $w$ of the form $\left(x_{1}, y_{1}, \ldots, x_{m}, y_{m}\right)$, satisfying the conditions: $x_{i} \in P_{1,2}-P_{1}$ and $y_{i} \in L-P_{1}$ for all $i, 1 \leq i \leq m$, and $|w|=x_{1} y_{1} \cdots x_{m} y_{m}=1$. (For any word $w_{0}$ in the elements of $G$, let $\left|w_{0}\right|$ denote the product of the entries of $w_{0}$, in the given 
order). Now, since $L=P_{1} *_{B} P_{3}$ is a free amalgamated product, there exists words

$$
w_{i}=\left(a_{1}^{(i)}, b_{1}^{(i)}, \cdots, a_{r_{i}}^{(i)}, b_{r_{i}}^{(i)}\right), \quad 1 \leq i \leq m,
$$

with $\left|w_{i}\right|=y_{i}$, and with

$$
\begin{aligned}
& a_{j}^{(i)} \in P_{3}-B, \quad 1 \leq j \leq r_{i}, \\
& b_{j}^{(i)} \in P_{1}-B, \quad 1 \leq j \leq r_{i}-1, \quad \text { and } \\
& b_{r_{i}}^{(i)} \in\left(P_{1}-B\right) \cup\{1\} .
\end{aligned}
$$

Such a decomposition of $y_{i}$ is possible since we may assume that any leading element belonging to $P_{1}$ has already been absorbed into $x_{i}$. Put $w^{\prime}=\left(x_{1}\right) \circ$ $w_{1} \circ \cdots \circ\left(x_{m}\right) \circ w_{m}$; the word obtained by concatenating the singleton words $\left(x_{i}\right)$ with the words $w_{i}$, in the given order. We may then write

$$
w^{\prime}=w_{1}^{\prime} \circ \cdots \circ w_{\ell}^{\prime}
$$

where the following conditions hold:

(1) For each $k$, all entries of $w_{k}^{\prime}$ lie in $P_{1,2}$, or all entries of $w_{k}^{\prime}$ lie in $P_{2,3}$.

(2) $\ell$ is minimal for the condition (1).

Since $G=P_{1,2 * P_{2}} P_{2,3}$ is a free amalgamated product, and since $\left|w^{\prime}\right|=|w|=1$, we either have $\left|w_{k}^{\prime}\right| \in P_{1,2}$ for all $k$, or $\left|w_{k}^{\prime}\right| \in P_{2,3}$ for all $k$, and then (2) yields $k=1$. Since $a_{1}^{(1)} \in P_{3}-B=P_{3}-P_{1,2}$, it now follows that all entries of $w^{\prime}$ lie in $P_{2,3}$, rather than in $P_{1,2}$. Since $x_{i} \in P_{1,2}$ we then have $x_{i} \in P_{2}$ for all $i$, and since $b_{j}^{(i)} \in P_{1}$ we get $b_{j}^{(i)} \in B$, whence $r_{i}=1$ and $y_{i}=a_{1}^{(i)}$. Thus, we have shown that $w=\left(x_{1}, a_{1}^{(1)}, \ldots, x_{m}, a_{m}^{(m)}\right)$ corresponds to a cycle in $\Gamma_{2,3}$. This proves the lemma.

2.7 Proposition. Assume that $\theta_{1,3} \leq \pi / 5$, that $\mathscr{H}$ has the weak convergence property, and that $\ell(\mathscr{Y} \cap A(h)) \leq 2$ for all $h \in \mathscr{H}-K$. Then, for both $k=1$ and 3 , we have $\theta_{2, k}^{*}=\pi / 2$ if and only if $\theta_{2, k}=\pi / 2$.

Proof. We have $\theta_{2, k} \leq \theta_{2, k}^{*}$ by $(2.5)(\mathrm{d})$, and hence $\theta_{2, k}=\theta_{2, k}^{*}$ if $\theta_{2, k}=$ $\pi / 2$, by $(2.5)(\mathrm{a})$. Thus we may assume by way of contradiction to (2.7) that $\theta_{1,2}^{*}=\pi / 2$ and $\theta_{1,2}<\pi / 2$.

From $\theta_{1,2}^{*}=\pi / 2$ we know that there exist elements $x$ and $x^{\prime}$ of $L-P_{3} K$ and elements $y$ and $y^{\prime}$ of $P_{2,3}-P_{3}$ such that $x y x^{\prime} y^{\prime} \in\langle\mathscr{H}\rangle$. Set $g=x y x^{\prime} y^{\prime}$, put $\mathbf{Y}^{\prime}=\mathbf{Y} y^{\prime}$, and define vertices $u$ and $v$ of $\mathbf{X}$ by $u=P_{2,3} x^{-1}$ and $v=u g$. Thus $u \in V(\mathbf{Y})$ and $v \in V\left(\mathbf{Y}^{\prime}\right)$. Further, we assume that $x, y, x^{\prime}, y^{\prime}$ have been chosen so as to minimize the length of $g$ as a word in $\mathscr{H}$.

As in the proof of $(2.5)$, let $\mathbf{h}=\left(h_{0}, \ldots, h_{n}\right)$ be a $(g, u)$-sequence. Then $d(u, v) \geq d\left(u h_{0}, v\right)$ and it then follows from $(1.1)(\mathrm{c})$ that $\ell\left([u, v] \cap A\left(h_{0}\right)\right)$ $\geq \frac{1}{2} a\left(h_{0}\right)$. But $\theta_{1,3} \leq \pi / 5$ by hypothesis, so $a\left(h_{0}\right) \geq 10$ and hence $\ell\left([u, v] \cap A\left(h_{0}\right)\right) \geq 5$. Then either $\ell\left(\mathbf{Y} \cap A\left(h_{0}\right)\right)>2$ or $\ell\left(\mathbf{Y}^{\prime} \cap A\left(h_{0}\right)\right)>2$, and our hypothesis then yields $h_{0} \in \mathscr{K}$ or $h_{0} \in \mathscr{K}^{y^{\prime}}$.

Suppose first that $h_{0} \in \mathscr{K}$, and put $x_{0}=h_{0}^{-1} x$. Then $x_{0} y x^{\prime} y^{\prime}=h_{1} \cdots h_{n}$ is of smaller length than $g$, so we must have had $g=1$ to begin with. Now assume that $g \neq 1$, so that $h_{0} \in K^{y^{\prime}}$. Put $u_{1}=u h_{0}$. Then $\left[u_{1}, v\right] \subseteq \mathbf{Y} h_{0} \cup \mathbf{Y}^{\prime}$. If $n \geq 1$ we may then argue as above, obtaining $h_{1} \in \mathscr{K}^{h_{0}} \cup \mathscr{K}^{y^{\prime}}$. But $h_{1} \notin \mathscr{K}^{y^{\prime}}$ 
as otherwise $h_{0} h_{1} \in K^{y^{\prime}}$ and $\mathbf{h}$ is not a minimal-length word for $g$. Thus $h_{1} \in \mathscr{K}^{h_{0}}$. In this case we have $h_{0} h_{1}=\left(h_{0} h_{1} h_{0}^{-1}\right) h_{0}$ where $h_{0} h_{1} h_{0}^{-1} \in \mathscr{K}$. Setting $x_{1}=h_{0} h_{1} h_{0}^{-1} x$ we then have $x_{1} y x^{\prime} y^{\prime}=h_{0} h_{2} \cdots h_{n}$ of smaller length than $g$, so again $g$ was just the identity element to begin with.

We have shown that either $g=1$ or $g \in \mathscr{K}^{y^{\prime}}$. Assume the latter case. Then $g=\left(y^{\prime}\right)^{-1} h y^{\prime}$ for some $h \in \mathscr{K}$, and then $y^{\prime} x y x^{\prime} \in \mathscr{K}$. By suitably replacing $x^{\prime}$ we then obtain $y^{\prime} x y x^{\prime}=1$ and $g=1$ in any case. But this means that $\theta\left(G ; L, P_{2,3} ; P_{3}\right) \geq \pi / 2$, and then $\theta_{1,2} \geq \pi / 2$ by (2.6). Since $\theta_{1,2}<\pi / 2$, we have the desired contradiction at this point.

\section{TREES AND CHAMBER SYSTEMS}

We begin by reviewing some basic notions. (See [9].)

A chamber system $\mathscr{C}$ consists of a set Cham $\mathscr{C}$ of chambers together with a set $\left\{\sim_{i}\right\}_{i \in I}$ of equivalence relations on Cham $\mathscr{C}$, indexed by a non-empty set $I$. The relation $\sim_{i}$ is called $i$-adjacency, and $\mathscr{C}$ is said to be a chamber system over $I$. The rank of $\mathscr{C}$ is the cardinality of $I$.

A gallery in a chamber system $\mathscr{C}$ is a sequence $\gamma=\left(\mathbf{c}_{0}, \ldots, \mathbf{c}_{n}\right)$ of chambers such that any two successive chambers $\mathbf{c}_{j-1}$ and $\mathbf{c}_{j}$ are $i_{j}$-adjacent for some $i_{j} \in I$. The sequence $\left(i_{1}, \ldots, i_{n}\right)$ is the type of $\gamma$, and $\gamma$ will be said to be reduced if in both $\gamma$ and typ $(\gamma)$ successive terms are always distinct. For any non-empty subset $J$ of $I$ there is an equivalence relation $\sim J$ generated by $\left\{\sim_{i}\right\}_{i \in J}$, and the corresponding equivalence classes are called the $J$-residues of $\mathscr{C}$. These are themselves chamber systems over $J$.

A chamber system of rank 2 may be viewed as a bipartite graph whose edges are the chambers and whose vertices are the rank- 1 residues. Thus, the rank- 2 residues of any chamber system may be viewed in this way.

A morphism $\phi: \mathscr{C} \rightarrow \mathscr{D}$ of chamber systems over $I$ is a mapping $\phi$ on the underlying sets of chambers such that $\phi$ preserves $i$-adjacency for all $i \in I$. If $X$ is a group of automorphisms of $\mathscr{C}$, then $($ Cham $\mathscr{C}) / X$ inherits a natural structure from $\mathscr{C}$, and one obtains in this way a chamber system $\mathscr{C} / X$ over I.

If $X$ is a group, $D$ is a subgroup, and $\left\{X_{i}\right\}_{i \in I}$ is a non-empty family of subgroups containing $D$, then there is a chamber system over $I$ :

$$
\mathscr{C}=\mathscr{C}\left(X, D,\left\{X_{i}\right\}_{i \in I}\right) \text {, }
$$

given by Cham $\mathscr{C}=D / X$, with

$$
D x \sim_{i} D y \quad \text { if and only if } x y^{-1} \in X_{i} .
$$

Henceforth, let $\mathscr{C}$ be the fixed chamber system

$$
\mathscr{C}=\mathscr{C}\left(G, B,\left\{P_{1}, P_{2}, P_{3}\right\}\right)
$$

over $I=\{1,2,3\}$, obtained in connection with the triangle $(T)$ and the group $G$ of section 2 . Our aim is to set up a correspondence between $\mathscr{C}$ and the tree $\mathbf{X}$. There are only two tools to work with: one is the simultaneous embedding of $\mathbf{Y}$ in $\mathbf{X}$ as a subtree and of $\mathbf{Y}$ in $\mathscr{C}$ as a rank- 2 residue over $\{1,3\}$. The other is the mapping

$$
\phi: \text { Cham } \mathscr{C} \rightarrow \text { Edges } \mathbf{X}
$$

given by $B g \mapsto P_{2} g,(g \in G)$.

The following lemma requires no proof. 
3.1 Lemma. Let $\mathbf{c}$ and $\mathbf{d}$ be chambers of $\mathscr{C}$.

(a) $\phi(\mathbf{c})=\phi(\mathbf{d})$ if and only if $\mathbf{c} \sim_{2} \mathbf{d}$.

(b) If $\mathbf{c} \sim_{i} \mathbf{d}$ with $i=1$ or 3 , then $\phi(\mathbf{c})$ and $\phi(\mathbf{d})$ are edges of $\mathbf{X}$ having a common vertex $v$, where $v$ is a right coset of $P_{i, 2}$ in $G$.

Recall that, in the Introduction, the families $\mathbb{E}$ and $\mathbb{F}$ of chamber systems over $\{1,2,3\}$ were presented in Figures 1 and 2 . For any chamber system $\mathscr{D}$ over $I$ and any family $\mathbb{G}$ of chamber systems over $I$, we say that $\mathscr{D}$ is $\mathbb{G}$-free if there exists no injective morphism of any member of $\mathbb{G}$ into $\mathscr{D}$. If $\mathbb{G}$ consists of a single chamber system $\mathscr{G}$, we say that $\mathscr{D}$ is $\mathscr{G}$-free if $\mathscr{D}$ is $\mathbb{G}$-free.

3.2 Lemma. Assume that $\mathscr{C}$ is $\mathscr{E}_{1}$-free and let $g \in G-L$. Then $\mathbf{Y} \cap \mathbf{Y} g$, as a subtree of $\mathbf{X}$, contains no path of length 3 .

Proof. Suppose false, and view $\mathbf{Y}$ and $\mathbf{Y g}$ as $\{1,3\}$-residues in $\mathscr{C}$. These residues are distinct since the set-wise stabilizer in $G$ of the $\{1,3\}$-residue $\mathbf{Y}$ is just $L$. Thus, (Cham $\mathbf{Y}) \cap($ Cham $\mathbf{Y g})=\varnothing$. But (3.1) implies that there are galleries $\left(\mathbf{c}_{1}, \mathbf{c}_{2}, \mathbf{c}_{3}\right)$ in $\mathbf{Y}$ and $\left(\mathbf{d}_{1}, \mathbf{d}_{2}, \mathbf{d}_{3}\right)$ in $\mathbf{Y} g$ of type $(1,3)$, with $\mathbf{c}_{i} \sim_{2} \mathbf{d}_{i},(1 \leq i \leq 3)$. This is contrary to $\mathscr{C}$ being $\mathscr{E}_{1}$-free.

Remark. Recall that $a(h) \geq 4$ for all $h \in \mathscr{H}$, by (2.2). It follows that if $\mathscr{C}$ contains an isomorphic copy of $\mathscr{E}_{1}$, then so does $\mathscr{C} /\langle h\rangle$ for any $h \in \mathscr{H}$. Since $\mathscr{E}_{1}=\mathscr{F}_{1}$, and since we are going to be assuming that $\mathscr{C} /\langle h\rangle$ is $\mathbb{E}$-free (or $\mathbb{F}$-free) for all $h \in \mathscr{H}$, we may therefore assume henceforth that $\mathscr{C}$ is $\mathscr{E}_{1}$-free.

3.3 Corollary. For any $h \in \mathscr{H}$ there is a unique $\{1,3\}$-residue of $\mathscr{C}$ which is invariant under $\langle h\rangle$.

Proof. It suffices to show that $\mathscr{H} \cap L=K-\{1\}$. Suppose false and let $h \in$ $(\mathscr{H} \cap L)-K$. Then $h \in K^{g}$ for some $g \in G-L$, and hence $\mathbf{Y} g$ is $h$-invariant, where $\mathbf{Y} g \neq \mathbf{Y}$. By (2.2) and (1.1)(b) the axes of $h$ on $\mathbf{Y}$ and on $\mathbf{Y} g$ coincide, contrary to $(3.2)$.

For any $h \in \mathscr{H}$ we now define $A_{\mathscr{C}}(h)$ to be the subsystem of $\mathscr{C}$ given by the axis of $h$ in the unique $\{1,3\}$-residue invariant under $h$. Thus, $A_{\mathscr{C}}(h)$ is an "infinite gallery" of "type" $(\ldots, 1,3,1,3, \ldots)$.

3.4 Lemma. Let $v$ be a vertex of $\mathbf{X}$, and let $h_{0}, h_{1}, h_{2}$ be elements of $\mathscr{H}$, no two of which lie in the same $G$-conjugate of $K$. Suppose that $v=A\left(h_{0}\right) \cap$ $A\left(h_{1}\right) \cap A\left(h_{2}\right)$ and that each $A\left(h_{i}\right) \cap A\left(h_{j}\right)$ contains an edge, $(0 \leq i \neq j \leq 2)$. For each $i$, let $\gamma_{i}$ be the pre-image in $A_{\mathscr{C}}\left(h_{i}\right)$, via $\phi$, of the set of edges of $A\left(h_{i}\right)$ for which $v$ is a vertex. Then $\gamma_{0} \cup \gamma_{1} \cup \gamma_{2}$ is the set of chambers of a cyclic reduced gallery of length 6 .

Proof. Let $S t(v)$ denote the set of edges at $v$, and let $(i, j, k)$ be an ordering of $\{0,1,2\}$. The hypothesis implies that there are three distinct edges $e_{0}, e_{1}, e_{2} \in S t(v)$ such that $A\left(h_{i}\right) \cap A\left(h_{j}\right) \cap S t(v)=\left\{e_{k}\right\}$. Since $h_{i}$ and $h_{j}$ are not in the same $G$-conjugate of $K$, we have $A_{\mathscr{C}}\left(h_{i}\right) \cap A_{\mathscr{C}}\left(h_{j}\right)=\varnothing$. The result now follows from (3.1) and (3.3).

Our aim now is to put together a sort of dictionary which will allow us to view the families $\mathbb{E}$ and $\mathbb{F}$ in terms of $\mathbf{X}$.

Let $\mathbf{s}=\left(h_{0}, \ldots, h_{k}\right)$ be a sequence of elements of $\mathscr{H}$, and assume that $\mathbf{s}$ has the property that the uniquely-determined $h_{i}$-invariant $G$-translates $\mathbf{Y}_{i}$ of 
$Y$ are all distinct. (In other words, no two of the elements $h_{i}$ lie in a common conjugate of $K$ ). In what follows, we set $h=h_{0}$, and we will always have $1 \leq k \leq 3$. Let $\mathbf{s}=\left(h_{0}, \ldots, h_{k}\right)$ be a sequence of elements of $\mathscr{H}$, and assume that $\mathbf{s}$ has the property that the uniquely-determined, $h_{i}$-invariant $G$ translates $\mathbf{Y}_{i}$ of $\mathbf{Y}$ are all distinct. (In other words, no two of the elements $h_{i}$ lie in a common conjugate of $K$ ). In what follows, we set $h=h_{0}$, and we will always have $1 \leq k \leq 3$.

Let $D_{0}(\mathbf{s})$ be a segment of $A(h)$, satisfying

$$
D_{0}(\mathbf{s}) \subseteq \bigcup_{i=1}^{k}\left(A(h) \cap A\left(h_{i}\right)\right) .
$$

Then put

$$
D_{1}(\mathbf{s})=\bigcup_{0 \leq i \neq j \leq k}\left(A\left(h_{i}\right) \cap A\left(h_{j}\right)\right),
$$

and let $D(\mathbf{s})$ be the subgraph of $D_{1}(\mathbf{s})$ consisting of $D_{0}(\mathbf{s})$ together with all the edges $e$ of $D_{1}(\mathbf{s})$ such that exactly one vertex of $e$ is in $A(h)$.

When drawing pictures of the various graphs $D(\mathbf{s})$ that will concern us, $D_{0}(\mathbf{s})$ will be drawn horizontally, and the edges of $D(\mathbf{s})$ not in $D_{0}(\mathbf{s})$ will be drawn vertically. For further emphasis, the vertices of $D_{0}(\mathbf{s})$ will be drawn as darkened circles, and the vertices of $D(\mathbf{s})-D_{0}(\mathbf{s})$ will appear as open circles.

3.5 Proposition. Let $h=h_{0} \in \mathscr{H}$, and let $s=\left(h_{0}, \ldots, h_{k}\right)$ be a sequence of elements of $\mathscr{H}$, no two of which lie in the same conjugate of $K$.

(a) If $\mathscr{C} /\langle h\rangle$ is $\mathbb{E}$-free, then $(D(\mathbf{s}), a(h), k)$ is not in the list given by Figure 3 (page 4550).

(b) If $\mathscr{C} /\langle h\rangle$ is $\mathbb{F}$-free, then $(D(\mathbf{s}), a(h), k)$ is not in the list given by Figure 4 (page 4551).

Proof. We will prove (a) in some detail, after which (b) should remain as a straightforward exercise.

Assume that $\mathscr{C} /\langle h\rangle$ is $\mathbb{E}$-free, and suppose first that $(D(\mathbf{s}), a(h), k)$ has the label $\mathscr{E}_{1}$ in Figure 3 . Here $k=1$, so $\mathbf{s}=\left(h, h_{1}\right)$, and $D(\mathbf{s})=D_{0}(\mathbf{s}) \subseteq$ $A(h) \cap A\left(h_{1}\right)$. But $D(\mathbf{s})$ has length 3 , which violates (3.2) and the remark following (3.2).

Suppose $(D(\mathbf{s}), a(h), k)$ has the label $\mathscr{E}_{2}$. Then $k=1, \mathbf{s}=\left(h, h_{1}\right)$, $\ell\left(A(h) \cap A\left(h_{1}\right)\right)=2$, and $a(h)=4$. Since $A_{\mathscr{C}}(h) \cap A_{\mathscr{C}}\left(h_{1}\right)=\phi$, by (3.3), it then follows from $(3.1)$ that $\mathscr{C} /\langle h\rangle$ contains a copy of $\mathscr{E}_{2}$.

Suppose $(D(\mathbf{s}), a(h), k)$ has the label $\mathscr{E}_{2}^{\prime}$. Then $\mathbf{s}=\left(h, h_{1}, h_{2}\right), a(h)=4$, and $D(\mathbf{s}) \neq D_{0}(\mathbf{s})$. The definition of $D(\mathbf{s})$ then says that $A\left(h_{i}\right) \cap A\left(h_{j}\right)$ is at least an edge, for all $i$ and $j, 0 \leq i \neq j \leq 2$. Then (3.1) and (3.4) together show that $\mathscr{C} /\langle h\rangle$ contains a copy of $\mathscr{E}_{2}^{\prime}$.

Suppose $(D(\mathbf{s}), a(h), k)$ is labelled by $\mathscr{E}_{3}$. Again $k=2$, and we are back to having $D(\mathbf{s})=D_{0}(\mathbf{s})$. Then $D(\mathbf{s})=\left(A(h) \cap A\left(h_{1}\right)\right) \cup\left(A(h) \cap A\left(h_{2}\right)\right)$ and (3.2) says that, up to symmetry, $A(h) \cap A\left(h_{1}\right)$ is the initial segment of length 2 in $D(\mathbf{s})$, and $A(h) \cap A\left(h_{2}\right)$ is the terminal length-2 segment in $D(\mathbf{s})$. Further, we are given $a(h) \leq 6$. If $a(h)=4$, then $\left(h, h_{1}\right)$ leads us back to $\mathscr{E}_{2}$, so we may assume $a(h)=6$. Then $(3.1)$ shows that $\mathscr{C} /\langle h\rangle$ contains a copy of $\mathscr{E}_{3}$.

The cases $\mathscr{E}_{3}^{\prime}, \mathscr{E}_{4}, \mathscr{E}_{4}^{\prime}$, and $\mathscr{E}_{5}$ are all very similar, so we shall jump ahead and consider only the case where $(D(\mathbf{s}), a(h), k)$ is labelled by $\mathscr{E}_{5}$. This means 

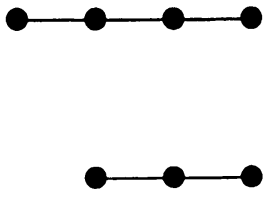

4

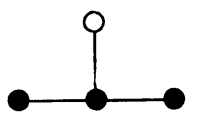

4

6
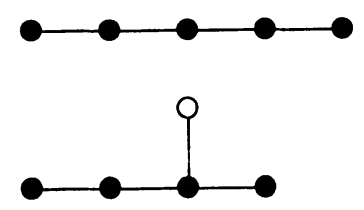

2

6

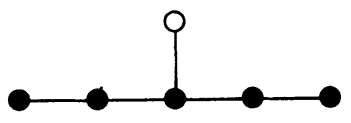

8
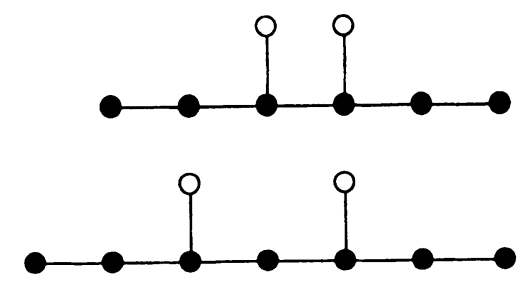

$\mathscr{E}_{1}$

$\mathscr{E}_{2}$

$\mathscr{E}_{3}$

$\mathscr{E}_{2}^{\prime}$

$\mathscr{E}_{3}^{\prime}$

$\mathscr{E}_{4}$

$\mathscr{E}_{4}^{\prime}$

$\mathscr{E}_{5}$

Figure 3. $\mathbb{E}$ as seen in $\mathbf{X}$

that $k=3, a(h) \leq 10$, and for a suitable ordering of the indices, the segments $A(h) \cap A\left(h_{1}\right), A(h) \cap A\left(h_{2}\right)$, and $A(h) \cap A\left(h_{3}\right)$ are successive segments of $D_{0}(\mathbf{s})$ of length 2 . Further, $A\left(h_{1}\right) \cap A\left(h_{2}\right)$ contains at least one edge, as does $A\left(h_{2}\right) \cap$ $A\left(h_{3}\right)$. If $a(h)=8$, then $\left(h, h_{1}, h_{2}\right)$ yyields the label $\mathscr{E}_{4}$, while if $a(h)=6$, then $\left(h, h_{1}, h_{2}\right)$ and a segment of length three from $D_{0}(\mathbf{s})$ yield the label $\mathscr{E}_{3}^{\prime}$. If $a(h)=4$, then $\left(h, h_{1}\right)$ brings us back to the case $\mathscr{E}_{2}$, so we may assume $a(h)=10$. Then $\mathscr{C} /\langle h\rangle$ contains the "10-gon" $A_{\mathscr{C}}(h) /\langle h\rangle$, while the segments $D(\mathbf{s}) \cap A\left(h_{1}\right), D(\mathbf{s}) \cap A\left(h_{2}\right)$, and $D(\mathbf{s}) \cap A\left(h_{3}\right)$ lift to subgalleries of $A_{\mathscr{C}}\left(h_{1}\right)$, $A_{\mathscr{C}}\left(h_{2}\right)$, and $A_{\mathscr{C}}\left(h_{3}\right)$ of lengths 3,4 , and 3 , respectively. From (3.1) and (3.4) we then get a copy of $\mathscr{E}_{5}$ in $\mathscr{C} /\langle h\rangle$.

We now leave case (b) to the reader. We remark that the reason that Figure 4 includes no label $\mathscr{F}_{2}$ is that the requirement that $\mathscr{C} /\langle h\rangle$ be $\mathscr{F}_{2}$-free is equivalent to the requirement that $a(h)$ be bigger than 4 . This has nothing to do with the way axes intersect in $\mathbf{X}$. 


\begin{tabular}{|c|c|c|c|}
\hline$k$ & $a\left(h_{0}\right) \leq$ & picture & label \\
\hline 1 & arbitrary & & $\mathscr{F}_{1}$ \\
\hline 0 & 4 & DNE) & $\mathscr{F}_{2}$ \\
\hline 1 & 6 & & $\mathscr{F}_{3}$ \\
\hline 3 & 6 & & $\mathscr{F}_{3}^{\prime}$ \\
\hline 2 & 8 & & $\mathscr{F}_{4}$ \\
\hline 3 & 8 & & $\mathscr{F}_{4}^{\prime}$ \\
\hline 3 & 10 & & $\mathscr{F}_{5}$ \\
\hline 3 & 10 & & $\mathscr{F}_{5}^{\prime}$ \\
\hline 3 & 12 & & $\mathscr{F}_{6}$ \\
\hline
\end{tabular}

Figure 4. $\mathbb{F}$ as seen in $\mathbf{X}$

\section{THEOREMS A AND B}

We continue now with the set-up of section 2 .

An $\mathscr{H}$-sequence is always a finite sequence of elements of $\mathscr{H}$. If $\mathbf{h}$ and $\mathbf{h}^{\prime}$ are $\mathscr{H}$-sequences, then $\mathbf{h} \circ \mathbf{h}^{\prime}$ is the $\mathscr{H}$-sequence obtained by concatenating $\mathbf{h}$ and $\mathbf{h}^{\prime}$ in the given order. If $\mathbf{h}=\left(h_{0}, \ldots, h_{n}\right)$ is an $\mathscr{H}$-sequence, then $\mathbf{h}^{-1}$ is the $\mathscr{H}$-sequence $\left(h_{n}^{-1}, \ldots, h_{0}^{-1}\right)$, and if $g \in\langle\mathscr{H}\rangle$, then $\mathbf{h}^{g}$ is the $\mathscr{H}$-sequence $\left(g^{-1} h_{0} g, \ldots, g^{-1} h_{n} g\right)$.

Let $\mathbf{h}$ and $\mathbf{h}^{\prime}$ be two $\mathscr{H}$-sequences of the same length:

$$
\mathbf{h}=\left(h_{0}, \ldots, h_{n}\right), \quad \mathbf{h}^{\prime}=\left(h_{0}^{\prime}, \ldots, h_{n}^{\prime}\right) .
$$

We say that $\mathbf{h}^{\prime}$ is a simple braiding of $\mathbf{h}$ if for some $i, 1 \leq i \leq n$, we have $h_{j}^{\prime}=h_{j}$ for all $j, j \notin\{i-1, i\}$, and

$$
\left(h_{i-1}^{\prime}, h_{i}^{\prime}\right)=\left(h_{i-1} h_{i} h_{i-1}^{-1}, h_{i-1}\right) \text { or }\left(h_{i}, h_{i}^{-1} h_{i-1} h_{i}\right)
$$


We say that $\mathbf{h}^{\prime}$ is a braiding of $\mathbf{h}$ if there exists a sequence

$$
\mathbf{h}=\mathbf{b}_{0}, \ldots, \mathbf{b}_{\ell}=\mathbf{h}^{\prime}
$$

of $\mathscr{H}$-sequences, with $\mathbf{b}_{j}$ a simple braiding of $\mathbf{b}_{j-1},(1 \leq i \leq \ell)$.

We recall from the Introduction that $\mathscr{l}^{*}$ denotes the set of all $\mathscr{H}$-sequences $\mathbf{h}=\left(h_{0}, \ldots, h_{n}\right)$ such that $\mathbf{h}$ is a word of minimal length for the element $h_{0} \cdots h_{n}$, in terms of $\mathscr{H}$.

\subsection{Lemma. The following hold.}

(a) If $\mathbf{h} \in \mathscr{H}^{*}$, then any braiding of $\mathbf{h}$ is in $\mathscr{H}^{*}$.

(b) If $\mathbf{h}=\left(h_{0}, \ldots, h_{n}\right)$ and $\mathbf{h}^{\prime}=\left(h_{0}^{\prime}, \ldots, h_{n}^{\prime}\right)$ is a braiding of $\mathbf{h}$, then $h_{0} \cdots h_{n}=h_{0}^{\prime} \cdots h_{n}^{\prime}$.

(c) If $\mathbf{h}=\left(h_{0}, \ldots, h_{n}\right) \in \mathscr{H}^{*}$, then for all $i$ and $j$ with $i \neq j$, we have $h_{i} h_{j} \notin \mathscr{H} \cup\{1\}$.

Proof. See [3, Lemmas 1.2 and 2.2] for the details.

4.2 Deiinition. A polarization (of $\mathbf{X}$ over $\mathscr{H}$ ) is a mapping $\mathscr{P}$ which associates to any $\mathbf{h} \in \mathscr{H}^{*}$ a collection $\mathscr{P}(\mathbf{h})$ of non-degenerate, oriented, closed segments of $\mathbf{X}$, in such a way that the following three conditions are satisfied.

(a) If $[u, v] \in \mathscr{P}(\mathbf{h})$, then $[v, u] \in \mathscr{P}\left(\mathbf{h}^{-1}\right)$.

(b) If $[u, v] \in \mathscr{P}(\mathbf{h})$ and $g \in\langle\mathscr{H}\rangle$, then $[u g, v g] \in \mathscr{P}\left(\mathbf{h}^{g}\right)$.

(c) If $\mathbf{h}^{\prime}$ is a braiding of $\mathbf{h}$, then $\mathscr{P}(\mathbf{h})=\mathscr{P}\left(\mathbf{h}^{\prime}\right)$.

Define subsets $\mathscr{H}_{1}$ and $\mathscr{H}_{2}$ of $\mathscr{H}$ as follows. If $\mathscr{C} \mid\langle h\rangle$ is $\mathbb{F}$-free for all $h \in K$, put $\mathscr{H}_{1}=\varnothing$ and $\mathscr{H}_{2}=\mathscr{H}$. Otherwise, we assume that $\mathscr{C} /\langle h\rangle$ is $\mathbb{E}$-free for all $h \in K$, and we put $\mathscr{H}_{1}=\mathscr{H}$ and $\mathscr{H}_{2}=\varnothing$.

4.3 Definition. Let $\mathscr{P}$ be a polarization of $\mathbf{X}$ over $\mathscr{H}$. We say that $\mathscr{P}$ controls convergence relative to $\left(\mathscr{H}_{1}, \mathscr{H}_{2}\right)$ if the following hold.

(a) (Existence). Whenever $h \in \mathscr{H}$ and $[u, v]$ is a segment of $A(h)$, we have

$$
[u, v] \in \mathscr{P}(h) \text { if } \begin{cases}\frac{1}{2} a(h)<d(u, v) \leq a(h) & \left(\text { for } \mathscr{H}=\mathscr{H}_{1}\right), \\ \frac{1}{2} a(h) \leq d(u, v) \leq a(h) & \text { (for } \left.\mathscr{H}=\mathscr{H}_{2}\right) .\end{cases}
$$

(b) (Exclusion). Whenever $\mathbf{h} \in \mathscr{H}^{*}, J \in \mathscr{P}(\mathbf{h})$, and $h \in \mathscr{H}$ with $\mathbf{h} \circ(h) \in$ $\mathscr{H}^{*}$, we have the following.
(i) $\ell(A(h) \cap J) \begin{cases}<\frac{1}{2} a(h) & \left(\text { for } \mathscr{H}=\mathscr{H}_{1}\right), \\ \leq \frac{1}{2} a(h) & \left(\text { for } \mathscr{H}=\mathscr{H}_{2}\right) .\end{cases}$

(ii) If $A(h) \cap J$ contains an end-point of $J$ then $\ell(A(h) \cap J) \leq \frac{1}{3} a(h)$. (iii) $J \nsubseteq A(h)$.

(c) (Extension). Whenever $\mathbf{h} \in \mathscr{H}^{*}, J=[u, v] \in \mathscr{P}(\mathbf{h}), h \in \mathscr{H}$ with $\mathbf{h} \circ(h) \in \mathscr{H}^{*}$, and $x$ is a point with $u$ in the open interval $(x, v)$ and with $[x, u] \subseteq A(h)$, then $[x h, v] \in \mathscr{P}(\mathbf{h} \circ(h))$ provided that

$$
\begin{array}{ll}
d(x, u)<\frac{1}{2} a(h) \leq d(x, u)+\ell(A(h) \cap J) & \left(\text { for } \mathscr{H}=\mathscr{H}_{1}\right), \\
d(x, u) \leq \frac{1}{2} a(h)<d(x, u)+\ell(A(h) \cap J) & \left(\text { for } \mathscr{H}=\mathscr{H}_{2}\right) .
\end{array}
$$

In the present context, the main theorem (Theorem 1.7) of [3] may now be stated as: 


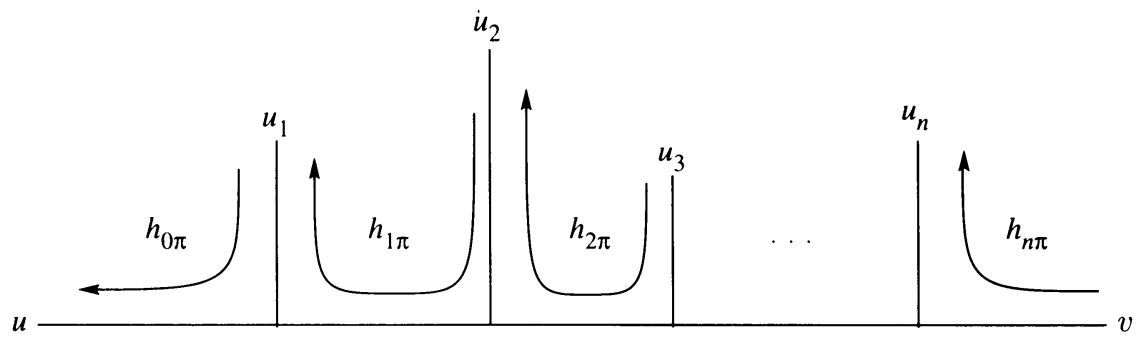

4.4 Theorem. Suppose that there exists a polarization $\mathscr{P}$ of $\mathbf{X}$ over $\mathscr{H}$, such that $\mathscr{P}$ controls convergence relative to $\left(\mathscr{H}_{1}, \mathscr{H}_{2}\right)$. Then $\mathscr{H}$ has the weak convergence property if $\mathscr{H}=\mathscr{H}_{1}$, and $\mathscr{H}$ has the convergence property if $\mathscr{H}=\mathscr{H}_{2}$.

The next item of business is to set up a suitable polarization $\mathscr{P}$. To do this, we have to recall two more notions from [3]. Namely, for any set $S$ of vertices of $\mathscr{X}$, the simplex spanned by $S$ is

$$
\Sigma(S)=\bigcup\{[u, v]: u, v \in S\},
$$

and we say that $S$ is in general position if $u \notin \Sigma(S-\{u\})$, for every $u \in S$.

We may now define a certain polarization $\mathscr{P}$. Namely, if $\mathbf{h}^{\prime} \in \mathscr{L}^{*}$ and $[u, v]$ is a segment of $\mathscr{X}$, we declare $[u, v]$ to be a member of $\mathscr{P}\left(\mathbf{h}^{\prime}\right)$ if there exists

- a braiding $\mathbf{h}=\left(h_{0}, \ldots, h_{n}\right)$ of $\mathbf{h}^{\prime}$,

- a permutation $\pi$ of $\{0, \ldots, n\}$, and

- a set $S=\left\{u_{0}, \ldots, u_{n+1}\right\}$ of vertices in general position, with $u=u_{0}$ and $v=u_{n+1}$,

such that the following three conditions hold for all $i, 0 \leq i \leq n$.

$(\mathscr{P} 1) \quad A\left(h_{i \pi}\right) \cap \Sigma(S)=\left[u_{i}, u_{i+1}\right]$ and

$$
d\left(u_{i}, u_{i+1}\right) \begin{cases}>\frac{1}{2} a\left(h_{i \pi}\right) & \text { if } \mathscr{H}=\mathscr{H}_{1}, \\ \geq \frac{1}{2} a\left(h_{i \pi}\right) & \text { if } \mathscr{H}=\mathscr{H}_{2} .\end{cases}
$$

$(\mathscr{P} 2) \quad A\left(h_{i}\right) \cap[u, v]$ is non-degenerate.

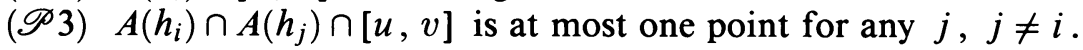

It is a straightforward task to check that $\mathscr{P}$ is a polarization of $\mathbf{X}$ over $\mathscr{H}$, in the sense of (4.2). Our aim will now be to show that $\mathscr{P}$ controls convergence relative to $\left(\mathscr{H}_{1}, \mathscr{H}_{2}\right)$.

4.5 Lemma. The polarization $\mathscr{P}$, defined above, satisfies the "existence" condition (4.3)(a).

Proof. Let $h \in \mathscr{H}$ and let $[u, v]$ be a segment of $A(h)$ with $d(u, v) \geq \frac{1}{2} a(h)$, and with strict inequality if $\mathscr{H}=\mathscr{H}_{1}$. Evidently, the singleton sequence $(h)$ is in $\mathscr{H}^{*}$, and $(h)$ is the only braiding of $(h)$. As $a(h) \neq 0$, the set $\{u, v\}$ is in general position. The condition $(\mathscr{P} 1)$ is satisfied, due to the given condition on $d(u, v)$. Now $(\mathscr{P} 2)$ holds since $[u, v] \subseteq A(h)$; and $(\mathscr{P} 3)$ is vacuously true. This yields the lemma.

We will now set up some notation to take us through the remaining steps in proving that $\mathscr{P}$ controls convergence relative to $\left(\mathscr{H}_{1}, \mathscr{H}_{2}\right)$. 
Fix $\mathbf{h}=\left(h_{0}, \ldots, h_{n}\right) \in \mathscr{H}^{*}$, and a segment $J=[u, v]$ in $\mathscr{P}(\mathbf{h})$. Let $\pi$ be a permutation of $\{0,1, \ldots, n\}$ and let $S=\left\{u_{0}, u_{1}, \ldots, u_{n+1}\right\}$ be a set of points in general position, with $u=u_{0}$ and $v=u_{n+1}$, and such that the conditions $(\mathscr{P} 1)$ through $(\mathscr{P} 3)$ hold with respect to $\mathbf{h}, J, \pi$, and $S$. Put $\Sigma=\Sigma(S)$, and put $h_{i}^{\prime}=h_{i \pi}$ for all $i, 0 \leq i \leq n$. Thus, $\left[u_{i}, u_{i+1}\right]$ is a segment of $A\left(h_{i}^{\prime}\right)$, by $(\mathscr{P} 1)$. Set $u=y_{0}$, and define points $y_{1}, \ldots, y_{n+1}$ by $\left[y_{i}, y_{i+1}\right]=J \cap A\left(h_{i}^{\prime}\right)$.

4.6 Lemma. Let $h \in \mathscr{H}$ with $\mathbf{h} \circ(h) \in \mathscr{H}^{*}$. Then $\left[u, y_{1}\right] \nsubseteq A(h)$ and $\left[y_{n}, v\right] \nsubseteq A(h)$.

Proof. Assume, by way of contradiction, that $\left[u, y_{1}\right] \subseteq A(h)$. As $\mathbf{h} \circ(h) \in \mathscr{H}^{*}$ we have $h_{0}^{\prime} h \notin \mathscr{H} \cup\{1\}$, by (4.1)(c). Then (3.2) implies that $d\left(u, y_{1}\right) \leq 2$.

Suppose first that $y_{1}=v$. That is, suppose that $n=0$ and that $J$ is a segment of $A\left(h_{0}\right)$. Then $\ell(J) \leq 2$, and $(\mathscr{P} 1)$ implies that $\mathscr{H}=\mathscr{H}_{2}, a\left(h_{0}\right)=$ 4 , and $\ell(J)=2$. But this is contrary to $\mathscr{C} /\left\langle h_{0}\right\rangle$ being $\mathscr{F}_{2}$-free. Thus, $y_{1} \neq v$, and $n \geq 1$.

Now $h_{0}^{\prime} h_{1}^{\prime} \notin \mathscr{H} \cup\{1\}$ by $(4.1)(\mathrm{c})$, and hence $d\left(y_{1}, u_{1}\right) \leq 2$. Thus, $d\left(u, u_{1}\right) \leq$ 4. Suppose $\mathscr{H}=\mathscr{H}_{1}$. Then $(\mathscr{P} 1)$ implies that either $a\left(h_{0}^{\prime}\right) \leq 6$ and $d\left(u, u_{1}\right)=$ 4 , or else $a\left(h_{0}^{\prime}\right)=4$ and $d\left(u, u_{1}\right)=3$. If $a\left(h_{0}^{\prime}\right)=6$, put $\mathbf{s}=\left(h_{0}^{\prime}, h_{1}^{\prime}, h\right)$ and observe that $D(\mathbf{s})$ is given by $\mathscr{E}_{3}$ in Figure 3. If $a\left(h_{0}^{\prime}\right)=4$, then either $d\left(u, y_{1}\right)=2$ or $d\left(y_{1}, u_{1}\right)=2$, and we take $\mathbf{s}=\left(h_{0}^{\prime}, h\right)$ or $\mathbf{s}=\left(h_{0}^{\prime}, h_{1}^{\prime}\right)$ accordingly. In this case, $D(\mathbf{s})$ is given by $\mathscr{E}_{2}$ in Figure 3 . We thus contradict $(3.5)(\mathrm{a})$, so $\mathscr{H}=\mathscr{H}_{2}$.

We now have either $a\left(h_{0}^{\prime}\right) \leq 8$ and $d\left(u, u_{1}\right)=4$, or $a\left(h_{0}^{\prime}\right) \leq 6$ and $d\left(u, u_{1}\right)=3$, or $a\left(h_{0}^{\prime}\right)=4$ and $d\left(u, u_{1}\right)=2$. In any case, with $\mathbf{s}$ as in the preceding paragraph, we see that $D(\mathbf{s})$ is in the list given by Figure 4 . This contradicts $(3.5)(\mathrm{b})$ and completes the proof of (4.6).

4.7 Lemma. The polarization $\mathscr{P}$ satisfies the "extension" condition (4.3)(c).

Proof. With $\mathbf{h}$ and $h$ as above, suppose that we are given a point $x$ with $u \in[x, v]$, such that $[x, u] \subseteq A(h)$ and such that:

$$
\begin{array}{ll}
d(x, u)<\frac{1}{2} a(h) \leq d(x, u)+\ell(A(h) \cap J) & \left(\text { if } \mathscr{H}=\mathscr{H}_{1}\right), \\
d(x, u) \leq \frac{1}{2} a(h)<d(x, u)+\ell(A(h) \cap J) & \left(\text { if } \mathscr{H}=\mathscr{H}_{2}\right) .
\end{array}
$$

Let $z$ be the nearest point on $J$ to $x h$. Then $[u, z]=A(h) \cap J$, and so $z \in\left[u, y_{1}\right)$, by (4.6). Put $u_{0}^{*}=x h$ and $u_{i+1}^{*}=u_{i}$ for $i=0, \ldots, n+1$; and put $\Sigma^{*}=\Sigma\left(\left\{u_{0}^{*}, \ldots, u_{n+2}^{*}\right\}\right)$. Then $\Sigma^{*}$ is the union of $\Sigma$ with $[x h, z]$. Let $\pi^{*}$ be the permutation of $\{0, \ldots, n+1\}$ which sends 0 to $n+1$ and which sends $i$ to $(i-1) \pi$ for $1 \leq i \leq n+1$. Put $h=h_{n+1}$ and $\mathbf{h}^{*}=\mathbf{h} \circ(h)$. We need only verify that the conditions $(\mathscr{P} 1)$ through $(\mathscr{P} 3)$ are in effect for $\mathbf{h}^{*}$, $J^{*}=[x h, v], \pi^{*}$, and $\Sigma^{*}$.

Condition $(*)$, above, guarantees that $\ell\left(\Sigma^{*} \cap A\left(h_{0 \pi^{*}}^{*}\right)\right)$ is large enough to satisfy $(\mathscr{P} 1)$ for $i=0$. For $i \geq 1$ we have $\left.\Sigma^{*} \cap A\left(h_{i \pi^{*}}^{*}\right)\right)=\Sigma^{*} \cap A\left(h_{(i-1) \pi}\right) \supseteq$ $\Sigma \cap A\left(h_{(i-1) \pi}\right)$, so the length of $\Sigma^{*} \cap A\left(h_{i \pi^{*}}^{*}\right)$ is large enough to satisfy $(\mathscr{P} 1)$ for all $i$. Further, since $z \in\left[u, y_{1}\right)$, it follows that $\Sigma^{*} \cap A\left(h_{i \pi^{*}}^{*}\right)=\left[u_{i}^{*}, u_{i+1}^{*}\right]$ for all $i$, and so $\left(\mathscr{P}_{1}\right)$ is established. Again, because $z \in\left[u, y_{1}\right),(\mathscr{P} 2)$ and (P3) follow. 
4.8 Lemma. If $u$ or $v$ is in $A(h)$, then $\ell(A(h) \cap J) \leq \frac{1}{3} a(h)$.

Proof. Suppose that $u \in A(h)$ and that $\ell(A(h) \cap J)>\frac{1}{3} a(h)$. By (4.1)(c), (3.2), and (4.6), we then have $\ell(A(h) \cap J)=2$ and $a(h)=4$. Then $(\mathscr{P} 1)$ implies that $\mathscr{H}=\mathscr{H}_{2}$. But then $\mathscr{C} \mid\langle h\rangle$ is $\mathscr{F}_{2}$-free, which means that $a(h)>4$; a contradiction.

4.9 Lemma. The polarization $\mathscr{P}$ satisfies the "exclusion" condition (4.3)(b).

Proof. By (4.6) and (4.8), it only remains to verify part (i) of (4.3)(b), and we may assume that $A(h) \cap J \subseteq(u, v)$. Put $A(h) \cap J=\left[x, x^{\prime}\right]$, with $d(u, x)<$ $d\left(u, x^{\prime}\right)$. Assume by way of contradiction that $d\left(x, x^{\prime}\right) \geq \frac{1}{2} a(h)$, with strict inequality if $\mathscr{H}=\mathscr{H}_{2}$.

Suppose first that for all $i, 1 \leq i \leq n$, we have $y_{i} \notin\left(x, x^{\prime}\right)$. This means that for some $j, 0 \leq j \leq n$, we have $\left[x, x^{\prime}\right] \subseteq A\left(h_{j}^{\prime}\right)$. Applying (4.1)(c) and (3.2), we get $d\left(x, x^{\prime}\right)=2, a(h)=4$, and $\mathscr{H}=\mathscr{H}_{1}$. Setting $\mathbf{s}=\left(h, h_{j}^{\prime}\right), D(\mathbf{s})$ is then $\mathscr{E}_{2}$ in Figure 3, and we contradict $(3.5)($ a).

We now have $y_{i} \in\left(x, x^{\prime}\right)$ for some $i$. Suppose next that neither $y_{i-1}$ nor $y_{i+1}$ is in $\left(x, x^{\prime}\right)$. Then $\left[x, x^{\prime}\right] \subseteq\left[y_{i-1}, y_{i+1}\right]$ and it follows that $d\left(x, x^{\prime}\right) \leq$ 4. Set $\mathbf{s}=\left(h, h_{i-1}^{\prime}, h_{i}^{\prime}\right)$, and suppose first that $\mathscr{H}=\mathscr{H}_{2}$. Then $a(h) \leq 6$, and then $a(h)=6$ since $\mathscr{C} /\langle h\rangle$ is then $\mathscr{F}_{2}$-free. Further, we have $\ell(A(h) \cap J)=4$, and then $D\left(\left(h, h_{i-1}^{\prime}\right)\right)$ is $\mathscr{F}_{3}$ in Figure 4, contrary to (3.5). Thus, $\mathscr{H}=\mathscr{H}_{1}$. If $a(h)=4$ take $D_{0}(\mathbf{s})$ to be the segment of $J$ of length 2 , with mid-point $y_{i}$. Then $D(\mathbf{s})$ is $\mathscr{E}_{2}^{\prime}$ in Figure 3. If $a(h)=6$, then $d\left(x, x^{\prime}\right) \geq 3$, and we may choose $D_{0}(\mathbf{s})$ of length 3 and obtain $D(\mathbf{s})$ as $\mathscr{E}_{3}^{\prime}$ in Figure 3. If $a(h)=8$, then $d\left(x, x^{\prime}\right)=4$ and we produce $\mathscr{E}_{4}$ in Figure 3 . In every case, (3.5) yields a contradiction.

We conclude that $\left[y_{i}, y_{i+1}\right] \subseteq\left(x, x^{\prime}\right)$ for some $i$. Put $\alpha=\left[u_{i}, y_{i}\right], \beta=$ $\left[y_{i}, y_{i+1}\right]$, and $\gamma=\left[y_{i+1}, u_{i+1}\right]$. Then $\alpha, \beta$, and $\gamma$ are non-degenerate, by definition of $\mathscr{P}$, and each has length at most 2 , by (3.2). Thus, $d\left(u_{i}, u_{i+1}\right) \leq$ 6 , and then $(\mathscr{P} 1)$ gives $a\left(h_{i}^{\prime}\right) \leq 12$, and $a\left(h_{i}^{\prime}\right) \leq 10$ if $\mathscr{H}=\mathscr{H}_{1}$.

Let $\lambda$ denote the sequence $(\ell(\alpha), \ell(\beta), \ell(\gamma))$. Assume $\mathscr{H}=\mathscr{H}_{1}$, and suppose first that $\lambda=(1,1,1)$. Then $d\left(u_{i}, u_{i+1}\right)=3$, so $a\left(h_{i}^{\prime}\right)=4$. Taking $\mathbf{s}=\left(h_{i}^{\prime}, h_{i-1}^{\prime}, h\right)$ and $D_{0}(\mathbf{s})=\alpha \circ \beta$, we find that $D(\mathbf{s})$ is $\mathscr{E}_{2}^{\prime}$ in Figure 3, contrary to (3.5). Suppose $\lambda=(1,1,2)$ or $(1,2,1)$. Then $a\left(h_{i}^{\prime}\right) \leq 6$ and we take $\mathbf{s}=\left(h_{i}^{\prime}, h, h_{i+1}^{\prime}\right)$ and $D_{0}(\mathbf{s})=\beta \circ \gamma$. This yields $\mathscr{E}_{3}^{\prime}$ in Figure 3. Suppose $\lambda=(1,2,2)$. Then $a\left(h_{i}^{\prime}\right) \leq 8$. Take $\mathbf{s}$ and $D_{0}(\mathbf{s})$ as in the preceding case and find that we have $\mathscr{E}_{4}$ in Figure 3. In the remaining two cases, where $\lambda=(2,1,2)$ and $a\left(h_{i}^{\prime}\right) \leq 8$, or $\lambda=(2,2,2)$ and $a\left(h_{i}^{\prime}\right) \leq 10$, take $\mathbf{s}=\left(h_{i}^{\prime}, h_{i-1}^{\prime}, h, h_{i+1}^{\prime}\right)$, and take $D_{0}(\mathbf{s})=\alpha \circ \beta \circ \gamma$. Observe that we then have $\mathscr{E}_{4}^{\prime}$ or $\mathscr{E}_{5}$ in Figure 3. Thus, in every case we contradict (3.5), and this proves (4.9) if $\mathscr{H}=\mathscr{H}_{1}$. The situation for $\mathscr{H}=\mathscr{H}_{2}$ is entirely similar and may safely be left to the reader.

4.10 Theorem B. (a) If $\mathscr{C} /\langle h\rangle$ is $\mathbb{E}$-free for all $h \in K$, then $\mathscr{H}$ has the weak convergence property.

(b) If $\mathscr{C} /\langle h\rangle$ is $\mathbb{F}$-free for all $h \in K$, then $\mathscr{H}$ has the convergence property.

Proof. Immediate from (4.4), (4.5), (4.7), and (4.9).

Theorem A is a corollary of $(4.10)(a),(3.2)$, and (2.4)(c). 


\section{THEOREMS C AND D}

We begin by proving Theorem $\mathrm{C}$. Thus, $\mathscr{C} /\langle h\rangle$ is assumed to be $\mathbb{E}$-free (or $\mathbb{F}$-free) for all $h \in K$. By (2.2), (3.2), and Theorem B, $\mathscr{H}$ satisfies hypothesis (2.3). Then by $(2.4)(\mathrm{c})$ the natural maps into colim $(T)$ are injective. Let $G^{*}$ be the colimit group given by $(2.4)(\mathrm{d})$, and identify each $P_{i, j}$ with its canonical image in $G^{*}$.

Let $\mathscr{C}^{*}$ denote the chamber system $\mathscr{C}\left(G^{*}, B,\left\{P_{i}\right\}_{i=1,2,3}\right)$. Each $\{i, j\}-$ residue of $\mathscr{C}^{*}$ is then isomorphic to a connected component of the graph $\Gamma_{i, j}=\Gamma\left(P_{i, j} ; P_{i}, P_{j} ; B\right)$, by $(2.5)(\mathrm{a})$. Thus, in order to complete the proof of Theorem $C$ it will suffice to prove:

5.1 Proposition. Let $\Sigma \in \mathbb{E} \cup \mathbb{F}$ and suppose that there is an injective morphism $\phi: \Sigma \rightarrow \mathscr{C}^{*}$ of chamber systems over $\{1,2,3\}$. Then there is also an injective morphism $\tilde{\phi}: \Sigma \rightarrow \mathscr{C} /\langle h\rangle$ for some $h \in K$.

Proof. As $\Sigma$ is connected, we may assume that $\operatorname{Im}(\phi)$ is contained in the connected component of $\mathscr{C}^{*}$ containing the chamber $B$. By $(2.4)(\mathrm{d})$ this component may be identified with a connected component of $\mathscr{C} /\langle\mathscr{H}\rangle$. Let $\pi$ denote the canonical projection of $\mathscr{C}$ onto $\mathscr{C} /\langle\mathscr{H}\rangle$.

Let $\Sigma_{0}, \ldots, \Sigma_{k}$ be the maximal reduced galleries in $\Sigma$ which are contained in $\{1,3\}$-residues of $\Sigma$. A glance at Figures 1 and 2 shows that the indices $0, \ldots, k$ can be chosen so that $\Sigma_{0}$ is 2 -adjacent to each $\Sigma_{i}$, and so that $\Sigma_{i}$ is not a cycle if $i>0$.

Let $\mathbf{Y}$ denote a $\{1,3\}$-residue of $\mathscr{C}$ such that $\phi\left(\Sigma_{0}\right) \subseteq \pi(\mathbf{Y})$. Without loss of generality, we may assume that $B$ is a chamber of $\mathbf{Y}$. Let $Z_{0}$ be a connected component of $\left(\left.\pi\right|_{\mathbf{Y}}\right)^{-1}\left(\phi\left(\Sigma_{0}\right)\right)$. If $\Sigma_{0}$ is a cycle, we then have $Z_{0}=A(h)$ for some $h \in K$, where $a(h)$ is the length of $\Sigma_{0}$. Otherwise, we have $\Sigma \cong \mathscr{E}_{1}$ and (in view of (2.2)) $Z_{0}$ is isomorphic to $\Sigma_{0}$. In this case we take $h=1$.

Let $\gamma=\left(c_{1}, \ldots, c_{\ell}\right)$ be a subgallery of $Z_{0}$ such that $\phi^{-1}(\pi(\gamma))$ is the subgallery of $\Sigma_{0}$ consisting of all chambers of $\Sigma_{0}$ which are 2-adjacent to chambers of $\Sigma-\Sigma_{0}$. Put $\mathbf{c}_{j}^{*}=\phi^{-1}\left(\pi\left(\mathbf{c}_{j}\right)\right)$ and let $\mathbf{d}_{j}^{*}$ be the chamber of $\Sigma-\Sigma_{0}$ to which $\mathbf{c}_{j}^{*}$ is 2 -adjacent. By $(2.5)(\mathrm{a})$ we can then find chambers $\mathbf{d}_{j}$ of $\mathscr{C}$ and galleries $Z_{j}$ in $\{1,3\}$-residues of $\mathscr{C}$ such that

(a) $\phi^{-1}\left(\pi\left(\mathbf{d}_{j}\right)\right)=\mathbf{d}_{j}^{*}$ and $\mathbf{c}_{j} \sim_{2} \mathbf{d}_{j}$,

(b) $\mathbf{d}_{j}$ is a chamber of $Z_{j}$, and

(c) $\pi\left(Z_{j}\right)=\phi\left(\Sigma_{i}\right)$ for some $i>0$.

Suppose that $j$ is an index such that $\mathbf{d}_{j}^{*}$ and $\mathbf{d}_{j+1}^{*}$ are $k$-adjacent for $k=1$ or 3 . There then exists $g \in\langle\mathscr{H}\rangle$ such that $d_{j} g$ and $d_{j+1}$ are $k$-adjacent. Then $\left(d_{j}, c_{j}, c_{j+1}, d_{j+1}, d_{j} g\right)$ is a gallery of type $(2, k, 2, k)$, and this means that, regarding $\mathbf{Y}$ as a subtree of $\mathbf{X}, g$ fixes a vertex of $\mathbf{Y}$. But $\langle\mathscr{H}\rangle$ acts freely on $\mathbf{X}$ by Theorem $\mathbf{B}$ (or by $(2.4)(\mathrm{b})$ ), so $g=1$ and $\mathbf{d}_{j} \sim_{k} \mathbf{d}_{j+1}$. In particular, it follows that $Z_{j}=Z_{j+1}$.

Suppose next that $\mathbf{d}_{j}^{*}$ and $\mathbf{d}_{j+1}^{*}$ are not $k$-adjacent in $\Sigma$, but that $\Sigma_{j}$ and $\Sigma_{j+1}$ are 2-adjacent at chambers $\mathbf{e}_{j}^{*}$ and $\mathbf{e}_{j+1}^{*}$. That is, we have a cyclic gallery $\left(\mathbf{e}_{j}^{*}, \mathbf{d}_{j}^{*}, \mathbf{c}_{j}^{*}, \mathbf{c}_{j+1}^{*}, \mathbf{d}_{j+1}^{*}, \mathbf{e}_{j+1}^{*}, \mathbf{e}_{j}^{*}\right)$ of length 6 , of type $(k, 2, k, 2, k, 2)$ where $k \in\{1,3\}$. The argument of the preceding paragraph may then be repeated, to show that $Z_{j}$ and $Z_{j+1}$ are 2 -adjacent at chambers $e_{j}$ and $e_{j+1}$, with $\pi\left(e_{j}\right)=\phi\left(e_{j}^{*}\right)$ and $\pi\left(e_{j+1}\right)=\phi\left(e_{j+1}^{*}\right)$. Thus $Z_{0} \cup \cdots \cup Z_{\ell}$ is a "lifting" of $\phi(\Sigma)$ into $\mathscr{C}$ which, modulo $\langle h\rangle$, yields an injective morphism of $\Sigma$. This result, completes the proof of 5.1 and of Theorem C. 
We next prove Theorem (0.3). Thus, suppose that the Stallings angles $\theta_{i, j}$ add up to at most $\pi,(1 \leq i<j \leq 3)$. If, say, $\theta_{1,3}=0$, then $K=1, \mathscr{H}=\phi$, and $(2.4)(\mathrm{d})$ provides a realization of $(T)$. So we may assume that $\theta_{i, j} \neq 0$ for all $i<j$. A glance through Figure 1 shows that $\mathscr{C} /\langle h\rangle$ is $\mathbb{E}$-free unless $\theta_{i, j}=\pi / 2$ and $\theta_{i, k} \geq \pi / 3$ for some choice of $\{i, j, k\}=\{1,2,3\}$. Assume $\theta_{1,2}=\pi / 2$ and $\theta_{1,3} \geq \pi / 3$. It then follows that $\theta_{2,3} \leq \pi / 6$. However, the largest cycle in any member of $\mathbb{E}$ is length 10 , so $\mathscr{\mathscr { C }} /\langle\bar{h}\rangle$ is $\mathbb{E}$-free. Now Theorem (0.3) follows from Theorem A.

We now come to the proof of Theorem $\mathrm{D}$. The following lemma will be needed.

5:2 Lemma. Let $(T)$ be any triangle of groups such that the natural maps into the colimit $G^{*}$ of $(T)$ are injective. Regard the vertex and edge-groups $P_{i, j}$ and $P_{i}$ as subgroups of $G^{*}$ and put $G_{0}=\left\langle P_{1}, P_{2}, P_{3}\right\rangle$ and $P_{i, j}^{0}=\left\langle P_{i}, P_{j}\right\rangle$. Then

$$
\theta\left(G^{*} ; P_{i, k}, P_{j, k} ; P_{k}\right)=\theta\left(G_{0} ; P_{i, k}^{0}, P_{j, k}^{0}, P_{k}\right)
$$

for any permutation $(i, j, k)$ of $\{1,2,3\}$.

Proof. It will suffice to take $(i, j, k)=(1,3,2)$. The subgroup of $G^{*}$ generated by $P_{1,2}$ and $P_{2,3}$ may be identified with the fundamental group of the following graph of groups (in which the associated monomorphisms are the obvious inclusion maps).

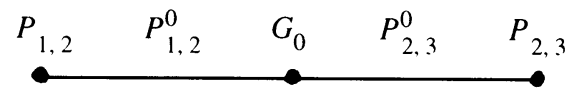

Suppose that we have a word $\mathbf{w}=\left(x_{1}, y_{1}, \ldots, x_{n}, y_{n}\right)$ for the identity element of $G^{*}$, with $x_{i} \in P_{1,2}-P_{2}$ and $y_{i} \in P_{2,3}-P_{2}$. We then view $\mathbf{w}$ as being a concatenation

$$
\mathbf{w}=\mathbf{w}_{1} \circ \cdots \circ \mathbf{w}_{k}
$$

where each $\mathbf{w}_{i}$ is a subsequence of $\mathbf{w}$, and where the product $g_{i}$ of the entries of $\mathbf{w}_{i}$ is an element of $P_{1,2} \cup G_{0} \cup P_{2,3}$. If necessary, we allow certain of the words $\mathbf{w}_{i}$ to be empty (and $g_{i}=1$ ), so that the sequence $\left(v_{1}, \ldots, v_{k}\right)$ of vertices of the graph $(*)$ given by $g_{i} \in G_{v_{i}}\left(=P_{1,2}, G_{0}\right.$, or $\left.P_{2,3}\right)$ is a "walk" in this graph. Moreover, we can do all this in such a way that $g_{1} \in G_{0}$, and so that $k$ is minimized subject to this last condition.

In this way we obtain a normal form $\left(g_{1}, \ldots, g_{k}\right)$ for the identity element of $G^{*}$ with respect to $(*)$, based at $G_{0}$. But [7, result 7.7] tells us that there is only one such normal form, and it is given by the sequence $\left(g_{1}=1\right)$ of length 1. Thus $\mathbf{w}=\mathbf{w}_{1}$ and we have $x_{i}, y_{i} \in G_{0}$ for all $i$. This proves the lemma.

5.3 Theorem D. If $(T)$ is non-spherical in the sense of $(0.2)$, then $\theta_{i, j}=\theta_{i, j}^{*}$ for all $i$ and $j, 1 \leq i<j \leq 3$.

Proof. By (5.2) we may assume without loss that each $P_{i, j}$ is generated by its subgroups $P_{i}$ and $P_{j}$. Suppose first that $\theta_{1,3}=0$. Then $K=1, \mathscr{H}=\varnothing$, $G=G^{*}$, and $\theta_{1,3}^{*}=0$. Moreover, (2.6) then yields $\theta_{2, k}^{*}=\theta_{2, k}$ for $k=1$ and 3 . (We remark that the proof of (2.6) in no way depends on the hypothesis (0.1), which is violated if $\theta_{i, j}=\pi$ for some $i \neq j$.) Thus, Theorem $\mathrm{D}$ holds if 
$\theta_{1,3}=0$. We are free to permute indices, so we may now assume that $\theta_{i, j}>0$ for all $i \neq j$. As $(T)$ is non-spherical, it then follows that $0<\theta_{i, j} \leq \pi / 2$ for all $i \neq j$, and at most one of the angles $\theta_{i, j}$ is $\pi / 2$.

Suppose next that $\{\pi / 2, \pi / 3\}$ is not contained in $\left\{\theta_{i, j} \mid 1 \leq i<j \leq 3\right\}$. Each even permutation of the indices 1,2, 3 leads to a different choice of $G$, $K$, and $\mathscr{C}$, and a glance at Figure 1 shows that for each such choice $\mathscr{C} /\langle h\rangle$ is $\mathscr{E}$-free for all $h \in K$. Theorem D then follows from $(2.5)(\mathrm{d})$ in this case. Thus, we may now assume that $\theta_{1,2}=\pi / 2$ and $\theta_{2,3}=\pi / 3$. As $(T)$ is non-spherical we then have $\theta_{1,3} \leq \pi / 6$. Theorem $\mathrm{D}$ now follows from $(2.5)(\mathrm{d})$ and (2.7). In detail: the hypotheses of (2.5) and (2.7) are fulfilled as a consequence of Theorem B and of (3.2). If $\theta_{2,3}^{*} \neq \pi / 3$, then $(2.5)(\mathrm{d})$ says that $\theta_{2,3}^{*}=\pi / 2$, contrary to (2.7). Thus $\theta_{2,3}^{*}=\pi / 3$, and (2.7) directly yields $\theta_{1,3}^{*}=\pi / 2$, as desired.

\section{REFERENCES}

1. R. Alperin and H. Bass, Length functions of group actions on $\Lambda$-trees, Combinatorial Group Theory and Topology (Alta, Utah, 1984), Ann. of Math. Studies, no. 111, Princeton Univ. Press, Princeton, NJ, 1987, pp. 265-378.

2. David Benson, Conway's group $\mathrm{CO}_{3}$ and the Dickson invariants, Preprint, 1993.

3. A. Chermak, $\mathbb{R}$-trees, small cancellation, and convergence, Trans. Amer. Math. Soc. 347 (1995), 4515-4531.

4. Colimits of sporadic triangles of groups, preprint, 1994.

5. Jon Corson, Complexes of groups, Proc. London Math. Soc. (3) 65 (1992), 199-224.

6. W.G. Dwyer and C.W. Wilkerson, A new finite loop space at the prime two, J. Amer. Math. Soc. 6 (1993), 37-64.

7. D.M. Goldschmidt, Graphs and groups, Groups and Graphs: New Results and Methods, Birkhäuser Verlag, Basel, 1985.

8. J. Morgan and P. Shalen, Valuations, trees, and degenerations of hyperbolic structures, I, Ann. of Math. (2) 120 (1984), 401-476.

9. Mark Ronan, Lectures on buildings, Academic Press, San Diego, 1989.

10. Ronald Solomon, Finite groups with Sylow 2-subgroups of type .3, J. Algebra 28 (1974), 182-198.

11. J. Stallings, Non-positively curred triangles of groups, Group Theory from a Geometrical Viewpoint, World Scientific, Singapore, 1991.

12. J. Tits, Sur le groupe des automorphismes d'un arbre, Essays on Topology and Related Topics, Mémoires dédiés à Georges de Rham, Springer-Verlag, Berlin and New York, 1970, pp. 188-211.

Department of Mathematics, Kansas State University, Manhattan, Kansas 66506

E-mail address: chermak@math.ksu.edu 